\title{
Expression of genes encoding the calcium signalosome in cellular and transgenic models of Huntington's disease
}

\author{
Magdalena Czeredys ${ }^{1}$, Joanna Gruszczynska-Biegala' ${ }^{1}$, Teresa Schacht ${ }^{2}$, Axel Methner ${ }^{2}$ and \\ Jacek Kuznicki ${ }^{1,3 *}$ \\ ${ }^{1}$ Laboratory of Neurodegeneration, International Institute of Molecular and Cell Biology, Warsaw, Poland \\ 2 Focus Program Translational Neuroscience, Rhine Main Neuroscience Network, Department of Neurology, Johannes Gutenberg University Medical Center Mainz, \\ Mainz, Germany \\ ${ }^{3}$ Laboratory of Calcium Binding Proteins, Nencki Institute of Experimental Biology, Warsaw, Poland
}

\section{Edited by:}

Gaiti Hasan, National Centre for

Biological Sciences, India

Reviewed by:

Nancy A. Muma, University of Kansas, USA

Ilya Bezprozvanny, UT Southwestern Medical Center at Dallas, USA

*Correspondence:

Jacek Kuznicki, Laboratory of Neurodegeneration, International Institute of Molecular and Cell

Biology, Trojdena 4, 02-109 Warsaw, Poland

e-mail: jkuznicki@iimcb.gov.pl
Huntington's disease (HD) is a hereditary neurodegenerative disease caused by the expansion of a polyglutamine stretch in the huntingtin (HTT) protein and characterized by dysregulated calcium homeostasis. We investigated whether these disturbances are correlated with changes in the mRNA level of the genes that encode proteins involved in calcium homeostasis and signaling (i.e., the calciosome). Using custom-made TaqMan low-density arrays containing probes for 96 genes, we quantified mRNA in the striatum in YAC128 mice, a model of HD, and wildtype mice. HTT mutation caused the increased expression of some components of the calcium signalosome, including calretinin, presenilin 2, and calmyrin 1, and the increased expression of genes indirectly involved in calcium homeostasis, such as huntingtin-associated protein 1 and calcyclin-binding protein. To verify these findings in a different model, we used PC12 cells with an inducible expression of mutated full-length HTT. Using single-cell imaging with Fura-2AM, we found that store-operated $\mathrm{Ca}^{2+}$ entry but not endoplasmic reticulum (ER) store content was changed as a result of the expression of mutant HTT. Statistically significant downregulation of the Orai calcium channel subunit 2, calmodulin, and septin 4 was detected in cells that expressed mutated HTT. Our data indicate that the dysregulation of calcium homeostasis correlates with changes in the gene expression of members of the calciosome. These changes, however, differed in the two models of HD used in this study. Our results indicate that each HD model exhibits distinct features that may only partially resemble the human disease.

Keywords: calcium signalosome, store-operated calcium entry, transgenic mice, TaqMan low-density arrays, Huntington's disease, huntingtin, huntingtin-associated protein 1, calcyclin-binding protein

\section{INTRODUCTION}

Cellular calcium transients control a vast array of cellular functions, from short-term responses (e.g., contraction and secretion) to the long-term regulation of cell growth and proliferation. Cytosolic $\mathrm{Ca}^{2+}$ increases in response to activation of cell-surface receptors, either directly after the activation of ionotropic glutamate channels or indirectly after generation of the $\mathrm{Ca}^{2+}$ mobilizing second messenger inositol 1,4,5-trisphosphate $\left(\mathrm{IP}_{3}\right)$. $\mathrm{IP}_{3}$ interacts with its receptors $\left(\mathrm{IP}_{3} \mathrm{R} 1-3\right)$ on the membrane of the endoplasmic reticulum (ER), the major store of $\mathrm{Ca}^{2+}$ in the cell. The opening of these $\mathrm{IP}_{3} \mathrm{R} \mathrm{Ca}^{2+}$ channels releases $\mathrm{Ca}^{2+}$ stored in the ER (Foskett et al., 2007). Increased cytosolic $\mathrm{Ca}^{2+}$ can also cause $\mathrm{Ca}^{2+}$-induced release of $\mathrm{Ca}^{2+}$ from ryanodine receptors (RyR1-3) (Hamilton, 2005). The depletion of $\mathrm{ER} \mathrm{Ca}^{2+}$ stores results in activation of store-operated channels (SOCs) at the plasma membrane, mediating store-operated calcium entry (SOCE) from the extracellular space, followed by the removal of cytosolic $\mathrm{Ca}^{2+}$ and replenishment of luminal $\mathrm{Ca}^{2+}$ through sarcoplasmic/ER $\mathrm{Ca}^{2+}$-adenosine triphosphatases (ATPases; SERCA1-3). Considering their electrophysiological and molecular properties, two main types of SOCs can be described.
The first are the highly $\mathrm{Ca}^{2+}$-selective calcium release activated calcium currents (ICRAC) mediated by the Orai family of proteins, which carry highly $\mathrm{Ca}^{2+}$-selective ICRAC currents (Vig et al., 2006). The second type of SOCs are non-selective $\mathrm{Ca}^{2+}$ permeable TRPC (C-type transient receptor potential) channels (Huang et al., 2006). The $\mathrm{Ca}^{2+}$ sensor's stromal interaction molecules 1 and 2 (STIM1 and STIM2) detect changes in $\mathrm{Ca}^{2+}$ concentration in the ER via an EF-hand $\mathrm{Ca}^{2+}$-binding domain, in response to store depletion, they rearrange into punctate structures that are close to the plasma membrane. This activates members of the Orai family (Orai1, Orai2, and Orai3) of $\mathrm{Ca}^{2+}$. influx channels (Liou et al., 2005; Roos et al., 2005), resulting in $\mathrm{Ca}^{2+}$ entry by ICRAC into the cell. STIM1 and Orail have also been implicated with cell death and mitochondrial bioenergetics (Henke et al., 2012, 2013). STIM1 and STIM2 are expressed in the brain (Klejman et al., 2009; Skibinska-Kijek et al., 2009; Ng et al., 2011; Steinbeck et al., 2011). The main function of STIM1 in neurons appears to be the activation of SOCE (Gruszczynska-Biegala et al., 2011). STIM1 directly suppresses the depolarizationinduced opening of the voltage-gated $\mathrm{Ca}^{2+}$ channel $\mathrm{Ca}_{V} 1.2$ by binding to the $\mathrm{C}$-terminus of this channel, leading to the 
inhibition of gating and long-term internalization of the channel from the membrane (Park et al., 2010). The primary function of STIM2 in neurons was suggested to be the regulation of the resting level of $\mathrm{Ca}^{2+}$ in the ER and $\mathrm{Ca}^{2+}$ leakage (GruszczynskaBiegala et al., 2011; Gruszczynska-Biegala and Kuznicki, 2013). To maintain the spatial and temporal properties of $\mathrm{Ca}^{2+}$ signals, neurons express numerous $\mathrm{Ca}^{2+}$-binding proteins that act as $\mathrm{Ca}^{2+}$ buffers (Nikoletopoulou and Tavernarakis, 2012). In the cytoplasm, parvalbumin (PV), calbindin D-28k (CALB1), and calretinin (CALB2) (Billing-Marczak and Kuznicki, 1999) act as $\mathrm{Ca}^{2+}$ buffers, whereas buffer proteins localized within the lumen of the ER, including calsequestrin (CASQ) and calreticulin (CALR) (Groenendyk et al., 2004; Michalak et al., 2009), allow the store to accumulate the large amounts of $\mathrm{Ca}^{2+}$ necessary for rapid cell signaling responses. These proteins involved in calcium signaling and homeostasis represent a special toolkit referred to as the $\mathrm{Ca}^{2+}$ signalosome (Berridge et al., 2003; Berridge, 2012).

Proper action of the $\mathrm{Ca}^{2+}$ signalosome is crucial for the functioning of neuronal cells and during aging the $\mathrm{Ca}^{2+}$ signaling machinery undergoes significant changes (Toescu and Verkhratsky, 2007; Puzianowska-Kuznicka and Kuznicki, 2009). Moreover, conclusive evidence indicates that neuronal $\mathrm{Ca}^{2+}$ signaling is abnormal in many neurodegenerative disorders (Wojda et al., 2008; Bezprozvanny, 2010). Notably, SOCE is dysregulated in epilepsy (Steinbeck et al., 2011) and AD (Jaworska et al., 2013; Ryazantseva et al., 2013). One of the neurodegenerative disorders with disrupted $\mathrm{Ca}^{2+}$ homeostasis is Huntington's disease (HD), which is characterized clinically by chorea, dementia, and psychiatric symptoms. This genetic disorder is caused by the expansion of a CAG trinucleotide repeat in exon 1 of the huntingtin gene (HTT), which is translated into polyglutamine residues (polyQ) in the huntingtin protein (HTT). Huntingtin is toxic to cells when the number of CAG repeats exceeds 36 , a point at which HTT forms aggregates in the nuclei of neurons where it might inhibit the function of various proteins, including key transcriptional factors. This leads to transcriptional dysfunction and may cause neuronal degeneration (Sugars and Rubinsztein, 2003; Li and $\mathrm{Li}, 2004)$. HD is characterized at the molecular level by various changes, including disturbances in calcium homeostasis and signaling components, and these changes are considered to be involved in various processes that lead to the neurodegeneration observed in HD (Giacomello et al., 2011, 2013).

It is probable that destabilization of neuronal $\mathrm{Ca}^{2+}$ signaling is one of the toxic functions of the mutant HTT. To test this hypothesis, we used two models of HD: a cellular model and a transgenic model overexpressing mutant HTT. Inducible PC12 cells overexpress an expanded HTT with 73 glutamines (Apostol et al., 2003) and are useful for studying early changes in HD, since they accumulate aggregates of mutant HTT upon treatment with the steroid ponasterone A. Although others have used this inducible PC12 model successfully to study HD pathogenesis, no disturbances in the expression or function of the calcium signalosome have yet been described. However, it was shown that the first 17 amino-acid residues of mutated HTT modulate its sub-cellular localization, aggregation and effects on $\mathrm{Ca}^{2+}$ homeostasis in glutamate-challenged PC12 cells (Rockabrand et al., 2007). Our HD animal model, YAC128 transgenic mice express the full-length human HTT protein with 128 CAG repeats and display an age-dependent loss of striatal neurons, similar to those seen in human HD (Slow et al., 2003). YAC128 mice initially exhibit hyperactivity, followed by the onset of motor deficits and finally hypokinesis. Motor deficits in YAC128 mice are highly correlated with striatal neuronal loss. There is compelling evidence that supports $\mathrm{Ca}^{2+}$ dysfunction playing a central role in the pathogenesis of HD. In medium spiny neurons (MSNs) from YAC128 transgenic mice but not control mice, the activity of SOCE was enhanced (Wu et al., 2011). Moreover, in a yeast twohybrid screen and YAC128 mice, an interaction between mutant HTT and the 5C-terminus of $\mathrm{IP}_{3} \mathrm{R} 1$ was shown to be mediated by huntingtin-associated protein-1A (Tang et al., 2003). Repeated application of glutamate to MSNs in these mice elevated cytosolic $\mathrm{Ca}^{2+}$ levels, resulting in apoptosis mediated by the activation of $\mathrm{G}_{q}$-coupled metabotropic glutamate receptors (Tang et al., 2005). Similar results were shown in the HdH (Q111/Q111) mouse model, in which agonist-induced $\mathrm{Ca}^{2+}$ release in neurons from these mice was increased relative to (Q20/Q20) control mice. Basal protein kinase $B(\mathrm{PKB})$ activation was also higher in $\mathrm{HdH}(\mathrm{Q} 111 / \mathrm{Q} 111)$ neurons, and this process was dependent on metabotropic glutamate receptor 5 (mGluR5) (Ribeiro et al., 2010).

Although the effect of mutant HTT on SOCE was previously studied in transgenic YAC128 mice (Wu et al., 2011), there is no data concerning calcium signalosome alterations, which could be considered to be involved in HD pathogenesis. Therefore, the aim of our study was to investigate whether mutated HTT is responsible for the changes in the expression of calciosome genes, and if so, which of such changes might explain the alterations observed in HD. Using custom-made TaqMan low-density arrays and individual real-time quantitative polymerase chain reaction (qRT-PCR), we studied changes in gene expression in two HD models. Our arrays contained most of the genes involved in calcium signaling and homeostasis, some genes responsible for amyloid beta precursor protein (APP) processing and insulin signaling, as well as a few genes that encode protein kinases or other proteins connected with HD. We found increased expression of few components of the calcium signalosome and genes indirectly involved in calcium homeostasis in YAC128 brains. In PC12 cells with induced expression of mutated full-length HTT, we detected downregulation of the genes that encode some proteins involved in the calcium signalosome. Our data indicate that the dysregulation of calcium homeostasis correlates with changes in the gene expression of members of the calciosome, but the two models used in this study exhibit distinct changes in gene expression.

\section{MATERIALS AND METHODS \\ ANIMALS}

Female transgenic YAC128 mice that overexpressed human HTT $(128 \mathrm{Gln})$ and non-transgenic littermates that were applied as controls aged between 3 and 6 months (Slow et al., 2003) were used. The animals were kept under normal laboratory conditions ( $12 \mathrm{~h} / 12 \mathrm{~h}$ dark/light cycle; $50-60 \%$ relative humidity; $22^{\circ} \mathrm{C}$ ) with food and water available ad libitum. Animal care was in accordance with the European Communities Council Directive (86/609/EEC). The experimental procedures were approved by 
the Local Commission for the Ethics of Animal Experimentation No. 1 in Warsaw (approval no. 305/212).

\section{EXAMINED BRAIN REGIONS}

The striatum (head of the caudate nucleus), the primary (M1) and secondary (M2) motor cortex, and the cerebellum were identified macroscopically according to anatomical landmarks (Paxinos and Franklin, 2004) in YAC128 and control mice brains. These brain structures were isolated and kept in $-80^{\circ} \mathrm{C}$ until the future experiments.

\section{CELL CULTURES}

PC12 cells with an inducible expression of full-length human HTT containing 73 polyglutamine repeats translated from a random codon with a TagRFP tag at the C-terminus were obtained from the Coriell Institute for Medical Research. The PC12 cells were grown to $90 \%$ confluence at $37^{\circ} \mathrm{C}$ in a $5 \% \mathrm{CO}_{2}, 96 \%$ relative humidity incubator in cell-culture plates $\left(100 \mathrm{~cm}^{2} ; \mathrm{BD}\right.$ Biosciences) treated with $10 \mu \mathrm{g} / \mu \mathrm{l}$ poly-L-lysine (Sigma) in a cell culture medium that contained 15\% horse serum (HS; H1270, Sigma), 2.5\% fetal bovine serum (FBS; F7524, Sigma), and phenol red-free Dulbecco's Modified Eagle Medium (DMEM; 41965-039, Gibco) supplemented with 1\% penicillin-streptomycin (P0781, Sigma), $0.1 \mathrm{mg} / \mathrm{ml}$ zeocin (R25001, Invitrogen), and $0.1 \mathrm{mg} / \mathrm{ml}$ geneticine (G8168, Sigma). The cells were passaged with a 1:5 dilution at least three times prior to the experiments. Each passage consisted of $5 \mathrm{~min}$ incubation with $2 \mathrm{ml}$ of trypsin replacement (Sigma), the addition of $8 \mathrm{ml}$ of cell media to inactivate the enzyme, and brief centrifugation $(2 \mathrm{~min}, 300 \times \mathrm{g}$ ), followed by media replacement. Cell suspensions were then added to the cellculture plates and pipetted into single cells. The cells grew to a monolayer ( $>50 \%$ confluence in 1-2 days), whereupon differentiation into neuron-like cells was achieved by treatment with neural growth factor (NGF 2.5S, $100 \mathrm{ng} / \mathrm{ml} ; 13257-019$, Invitrogen) for $48 \mathrm{~h}$ in low serum medium that contained DMEM, $1 \% \mathrm{HS}$, and $0.25 \%$ FBS supplemented by antibiotics as above. To induce the expression of human HTT, $2 \mu \mathrm{M}$ ponasterone A (P3490, Sigma) was added to the differentiated cells for $48 \mathrm{~h}$. As ponasterone A was dissolved in 100\% ethanol, control cells were treated with the same amount of $100 \%$ ethanol in parallel with the ponasterone A-treated cells.

\section{SINGLE-CELL Ca ${ }^{2+}$ MEASUREMENTS}

Single-cell $\mathrm{Ca}^{2+}$ imaging was performed using the ratiometric $\mathrm{Ca}^{2+}$ indicator dye Fura-2 acetoxymethyl ester (Fura-2 AM). The cells $(17,000$ cells/well) were differentiated into neuron-like cells in 96-well imaging plates (BD Bioscience) for $48 \mathrm{~h}$ and induced to express human HTT $48 \mathrm{~h}$ before the experiment. The cells were loaded with $5 \mu$ M Fura-2 AM (F1201, Molecular Probes) for $30 \mathrm{~min}$ at $37^{\circ} \mathrm{C}$ in Hank's Balanced Salt Solution supplemented with $2 \mathrm{mM} \mathrm{CaCl}_{2}$ and then rinsed and left undisturbed for $30 \mathrm{~min}$ to allow for de-esterification. Calcium-free medium instead of $2 \mathrm{mM} \mathrm{CaCl}_{2}$ contained $0.5 \mathrm{mM}$ ethylene glycol tetraacetic acid (EGTA). The experiments were performed using a BD Pathway 855 High Content Imaging System (BD Biosciences). Images were acquired at 340 and $380 \mathrm{~nm}$ excitation wavelengths, and the ratio was calculated every $5 \mathrm{~s}$ for every cell.

\section{RNA ISOLATION FROM BRAIN TISSUE AND PC12 CELLS}

Brain samples were homogenized separately using a PotterElvehjem homogenizer. RNA was extracted using the RNeasy Lipid Tissue Mini kit with additional DNase treatment (Qiagen). To isolate RNA from PC12 cells, homogenization was performed using Qiashredders (Qiagen), and RNA was extracted using the RNeasy Plus Mini kit (Qiagen). cDNA was synthesized by reverse transcription (SuperScript III RnaseH, Invitrogen).

\section{GENE EXPRESSION ANALYSIS}

All gene expression analysis were performed using the 7900HT system (Life Technologies). Gene profiling in the brain was performed using custom-designed TaqMan low-density arrays (Life Technologies), hereinafter referred to as RT-qPCR arrays. For each array (384 assays), $1.6 \mu \mathrm{g}$ of cDNA was loaded. The obtained data were analyzed using the relative quantification method and $2^{-\Delta \mathrm{CT}}$ formula $\left(\Delta \mathrm{CT}=\mathrm{CT}_{\text {target }}-\mathrm{CT}_{\text {Gapdh }}\right.$, where $\mathrm{CT}$ denotes the cycle threshold). The list of genes and their annotations included in the RT-qPCR arrays is shown in Table 1. For RTqPCR arrays TaqMan chemistry (4369016, Life Technologies) was used.

Single analyses of gene expression levels using cDNA preparations from PC12 cell cultures were performed in individual real-time PCR reactions, hereinafter referred to as qRT-PCR. The obtained data were quantified using the relative standard curve method. For each gene, standard cDNAs were diluted and amplified along with sample cDNAs in the same PCR run. Standard curves were generated using the 7900HT system software (Life Technologies). The quantity of mRNA in each sample $\left(\mathrm{R}_{0}\right)$ was determined from the relative standard curve (using sample CT values) and expressed in arbitrary units corresponding to the dilution factors of the standard RNA preparation. The amplification efficiency (E) of each PCR reaction was determined using the equation of the standard curve:

$$
\begin{array}{r}
\mathrm{CT}=-1 / \log (\mathrm{E}+1) \times \log \mathrm{Ro}+\log \mathrm{R} / \log (\mathrm{E}+1) \\
\mathrm{E}=10^{-1 / \text { Slope }}-1 ; \text { slope }=-1 / \log [\mathrm{E}+1] .
\end{array}
$$

Rat-specific primers were designed using Primer Blast (National Center for Biotechnology Information), and their sequences are shown in Table 2. For individual qRT-PCR in PC12 cells SYBR Green chemistry (4385612, Life Technologies) was used. The expression of the Tmem66, Cracr2a (Efcab4b), Ryr3, and Atp2al genes was verified using FAM dye-labeled TaqMan probes (Life Technologies) and TaqMan chemistry (4369016, Life Technologies). The expression of Cracr2a (Efcab4b), Ryr3, and Atp2a1 was undetectable in PC12 cells using both hand-made and custom-made primers.

\section{IMMUNOBLOTTING}

Cells were extracted in ice-cold RIPA buffer: $50 \mathrm{mM}$ Tris, $\mathrm{pH}$ 7.5, $150 \mathrm{mM} \mathrm{NaCl}, 1 \% \mathrm{NP}-40,0.5 \% \mathrm{NaDOC}, 0.1 \%$ sodium dodecyl sulfate (SDS), and $1 \mathrm{mM}$ ethylenediaminetetraacetic acid (EDTA) that contained mini complete protease inhibitor cocktail (Roche) and phosphatase inhibitors (Sigma). The lysed cells 
Table 1 | List of genes and their annotations included in the RT-qPCR array.

\begin{tabular}{|c|c|}
\hline Gene symbol & Gene name \\
\hline Actb & actin, beta \\
\hline Akt1 & thymoma viral proto-oncogene 1 \\
\hline Akt2 & thymoma viral proto-oncogene 2 \\
\hline Akt3 & thymoma viral proto-oncogene 3 \\
\hline Aph1a & $\begin{array}{l}\text { anterior pharynx defective 1a } \\
\text { homolog (C. elegans) }\end{array}$ \\
\hline Aph1b & $\begin{array}{l}\text { anteri or pharynx defective } 1 \mathrm{~b} \\
\text { homolog (C. elegans) }\end{array}$ \\
\hline Aph1c & $\begin{array}{l}\text { anterior pharynx defective 1c } \\
\text { homolog (C. elegans) }\end{array}$ \\
\hline Apoe & apolipoprotein E \\
\hline$A p p$ & amyloid beta precursor protein \\
\hline Atp2a1 & $\begin{array}{l}\text { ATPase, } \mathrm{Ca}^{2+} \text { transporting, } \\
\text { cardiac muscle, fast twitch } 1\end{array}$ \\
\hline Atp2a2 & $\begin{array}{l}\text { ATPase, } \mathrm{Ca}^{2+} \text { transporting, } \\
\text { cardiac muscle, slow twitch } 2\end{array}$ \\
\hline Atp2a3 & $\begin{array}{l}\text { ATPase, } \mathrm{Ca}^{2+} \text { transporting, } \\
\text { ubiquitous }\end{array}$ \\
\hline Atp2c1 & ATPase, $\mathrm{Ca}^{2+}$ sequestering \\
\hline Bace1 & beta-site APP cleaving enzyme 1 \\
\hline Cacna1a & $\begin{array}{l}\text { calcium channel, } \\
\text { voltage-dependent, P/Q type, } \\
\text { alpha } 1 \text { A subunit }\end{array}$ \\
\hline Cacna1b & $\begin{array}{l}\text { calcium channel, } \\
\text { voltage-dependent, } \mathrm{N} \text { type, alpha } \\
\text { 1B subunit }\end{array}$ \\
\hline Cacna1c & $\begin{array}{l}\text { calcium channel, } \\
\text { voltage-dependent, L type, alpha } \\
\text { 1C subunit }\end{array}$ \\
\hline Cacna1d & $\begin{array}{l}\text { calcium channel, } \\
\text { voltage-dependent, L type, alpha } \\
\text { 1D subunit }\end{array}$ \\
\hline Cacna1e & $\begin{array}{l}\text { calcium channel, } \\
\text { voltage-dependent, R type, alpha } \\
\text { 1E subunit }\end{array}$ \\
\hline Cacna1g & $\begin{array}{l}\text { calcium channel, } \\
\text { voltage-dependent, T type, alpha } \\
1 \mathrm{G} \text { subunit }\end{array}$ \\
\hline Cacna1h & $\begin{array}{l}\text { calcium channel, } \\
\text { voltage-dependent, T type, alpha } \\
1 \mathrm{H} \text { subunit }\end{array}$ \\
\hline Cacna1i & $\begin{array}{l}\text { calcium channel, } \\
\text { voltage-dependent, alpha 1I } \\
\text { subunit }\end{array}$ \\
\hline Cacybp & calcyclin binding protein \\
\hline Calb1 & calbindin 1 \\
\hline Calb2 & calbindin 2 \\
\hline Calm1 & calmodulin 1 \\
\hline Calr & calreticulin \\
\hline Camk2a & $\begin{array}{l}\text { calcium/calmodulin-dependent } \\
\text { protein kinase II alpha }\end{array}$ \\
\hline
\end{tabular}

\section{Assay ID}

Mm00607939_s1

Mm01331626_m1

Mm02026778_g1

Mm00442194_m1

Mm03647119_g1

Mm00781167_s1

Mm00503295_m1

Mm01307193_g1

Mm01344172_m1

Mm01275320_m1

Mm01201431_m1

Mm00443897_m1

Mm00723486_m1

Mm00478664_m1

Mm00432190_m1

Mm01333678_m1

Mm01188822_m1

Grm5

Mm01209919_m1

Gsk3a

Mm00494444_m1 Gusb

Hap 1

Hpca

Mm00486572_m1

Htr3a

Htt

lgfir

Mm01299033_m1

Insr

Irs1

Irs2

Mm01295897_g1

Mm00486647_m1

Mm00801461_m1

Mm01336281_g1

Mm00482936_m1

Mm00437967_m1

(Continued)

Itpr1

Itpr2
Table 1 | Continued

Gene symbol Gene name Assay ID

calnexin

calsequestrin 1

calsequestrin 2

calcium-sensing receptor

calpastatin

CD33 antigen

calcium and integrin binding 1

(calmyrin)

calcium and integrin binding

family member 2

cAMP responsive element

binding protein 1

CREB binding protein

drebrin 1

fibulin 1

forkhead box $\mathrm{O} 1$

forkhead box $\mathrm{O} 3$

glyceraldehyde 3-phosphate

dehydrogenase

guanine nucleotide binding protein, alpha q polypeptide

glutamate receptor, ionotropic, AMPA1 (alpha 1)

glutamate receptor, ionotropic, AMPA3 (alpha 3)

glutamate receptor, ionotropic, NMDA1 (zeta 1)

glutamate receptor, ionotropic, NMDA2A (epsilon 1)

glutamate receptor, metabotropic 5

glycogen synthase kinase 3 alpha

glycogen synthase kinase 3 beta

glucuronidase, beta

huntingtin-associated protein 1

hippocalcin

5-hydroxytryptamine (serotonin) receptor 3A

huntingtin

insulin-like growth factor 1

receptor

insulin receptor

insulin receptor substrate 1

insulin receptor substrate 2

inositol 1,4,5-trisphosphate

3-kinase $B$

inositol 1,4,5-trisphosphate

receptor 1

inositol 1,4,5-triphosphate

receptor 2

inositol 1,4,5-triphosphate

receptor 3
Mm00500330_m1

Mm00486733_m1

Mm00486742_m1

Mm00443375_m1

Mm00807001_m1

Mm00491152_m1

Mm00501944_m1

Mm00498053_m1

Mm00501607_m1

Mm01342452_m1

Mm00517314_m1

Mm00515700_m1

Mm00490672_m1

Mm01185722_m1

Mm99999915_g1

Mm00492381_m1

Mm00433753_m1

Mm00497506_m1

Mm00433790_m1

Mm00433802_m1

Mm00690332_m1

Mm01719731_g1

Mm00444911_m1

Mm01197698_m1

Mm00468825_m1

Mm00650703_m1

Mm00442874_m1

Mm01213820 m1

Mm00802831_m1

Mm01211875_m1

Mm01278327_m1

Mm03038438_m1

Mm01322781_m1

Mm00439907_m1

Mm00444937_m1

Mm01306070_m1 


\section{Table 1 | Continued}

\begin{tabular}{|c|c|}
\hline Gene symbol & Gene name \\
\hline Mtor & $\begin{array}{l}\text { mammalian target of } \\
\text { rapamycin (serine/threonine } \\
\text { kinase) }\end{array}$ \\
\hline Ncald & neurocalcin delta \\
\hline Ncs1 & $\begin{array}{l}\text { neuronal calcium } \\
\text { sensor } 1\end{array}$ \\
\hline Ncstn & nicastrin \\
\hline Orai1 & $\begin{array}{l}\text { ORAI calcium } \\
\text { release-activated calcium } \\
\text { modulator } 1\end{array}$ \\
\hline Orai2 & $\begin{array}{l}\text { ORAI calcium } \\
\text { release-activated calcium } \\
\text { modulator } 2\end{array}$ \\
\hline Orai3 & $\begin{array}{l}\text { ORAl calcium } \\
\text { release-activated calcium } \\
\text { modulator } 3\end{array}$ \\
\hline Pik3ca & $\begin{array}{l}\text { phosphatidylinositol 3-kinase, } \\
\text { catalytic, alpha polypeptide }\end{array}$ \\
\hline Plcb1 & phospholipase C, beta 1 \\
\hline Plcg 1 & phospholipase C, gamma 1 \\
\hline Рpp3ca & $\begin{array}{l}\text { protein phosphatase } 3, \\
\text { catalytic subunit, alpha } \\
\text { isoform }\end{array}$ \\
\hline Psen 1 & presenilin 1 \\
\hline Psen2 & presenilin 2 \\
\hline Psenen & $\begin{array}{l}\text { presenilin enhancer } 2 \\
\text { homolog (C. elegans) }\end{array}$ \\
\hline Pten & $\begin{array}{l}\text { phosphatase and tensin } \\
\text { homolog }\end{array}$ \\
\hline Pvalb & parvalbumin \\
\hline Rcvrn & recoverin \\
\hline Rgs4 & $\begin{array}{l}\text { regulator of G-protein } \\
\text { signaling } 4\end{array}$ \\
\hline Ryr1 & $\begin{array}{l}\text { ryanodine receptor } 1 \text {, skeletal } \\
\text { muscle }\end{array}$ \\
\hline Ryr2 & $\begin{array}{l}\text { ryanodine receptor } 2, \\
\text { cardiac }\end{array}$ \\
\hline Ryr3 & ryanodine receptor 3 \\
\hline S100a1 & $\begin{array}{l}\text { S100 calcium binding } \\
\text { protein A1 }\end{array}$ \\
\hline S100a6 & $\begin{array}{l}\text { S100 calcium binding protein } \\
\text { A6 (calcyclin) }\end{array}$ \\
\hline Sept4 & septin 4 \\
\hline Sirt1 & $\begin{array}{l}\text { sirtuin } 1 \text { (silent mating type } \\
\text { information regulation } 2, \\
\text { homolog) } 1 \text { (S. cerevisiae) }\end{array}$ \\
\hline Slc25a3 & $\begin{array}{l}\text { solute carrier family } 25 \\
\text { (mitochondrial carrier, } \\
\text { phosphate carrier) }\end{array}$ \\
\hline Stim1 & stromal interaction molecule 1 \\
\hline Stim2 & stromal interaction molecule 2 \\
\hline $\operatorname{Trpc1}$ & $\begin{array}{l}\text { transient receptor potential } \\
\text { cation channel, subfamily C, } \\
\text { member } 1\end{array}$ \\
\hline Vsn/1 & visinin-like 1 \\
\hline
\end{tabular}

Table 2 | List of primers used in individual qRT-PCR for PC12 cells.

\begin{tabular}{|c|c|c|c|}
\hline Assay ID & $\begin{array}{l}\text { PCR } \\
\text { amplicon }\end{array}$ & Forward primer & Reverse primer \\
\hline \multirow{2}{*}{ Mm00444968_m1 } & Sept4 & AGAGCATGACCCGGCTAGTA & GCCGCAGCTCTTCATCTTTC \\
\hline & Orai1 & AGAGCATGACCCGGCTAGTA & TGCCCGGTGTTAGAGAATGG \\
\hline Mm00774745_m1 & Orai2 & TCCATACTCCTGTCCTCGC & GGCCACGTGGTTGTGTTTTT \\
\hline \multirow{2}{*}{ Mm00490552_m1 } & Orai3 & GGTAACTATTCCCGCTGGCT & CAGCTACACCACAAACGCTG \\
\hline & Stim1 & CTGGAGAAGAAGCTGCGTGA & TTTTGGCGGCTCCTCTCATT \\
\hline \multirow{4}{*}{$\begin{array}{l}\text { Mm00452010_m1 } \\
\text { Mm00774349_m1 }\end{array}$} & Stim2 & TGTCTTTGCCATGGCTGGAT & СTTCTGTGGGCACACTCCAT \\
\hline & Ryr1 & GGACTACCTGTACATGGCTTAC & ССТСТТСТТСАССТССТТСТТС \\
\hline & Ryr2 & СТССТCACCTGGAAAGGATAAG & GTCATCTCTAACCGGACCATAC \\
\hline & Atp2c1 & TCATTCGAAAACCCCCTCGG & GAAGCTCTCGCCAGAAGACA \\
\hline \multirow{3}{*}{ Mm04214089_s1 } & Atp2a3 & CACAGTAGCCCGGAGGAGAA & TGTCACCGAGAAGCGACG \\
\hline & Atp2a2 & TCACACAAAGACCGTGGAGG & СTTCTTCAGCCGGCAATTCG \\
\hline & Itrp1 & TCTGGAAAGCTGCTAAGCCC & ATGACCGTCCCCAGCAATTT \\
\hline \multirow{3}{*}{ Mm01612888_m1 } & Itpr2 & GAGTCCAACCTCTTGAGCCC & TCCGGTAGTTGTTGCCCTTG \\
\hline & Itpr3 & CGTCATGAACCACGGACTGA & ACTCGTCTTTGGAGGGCTTG \\
\hline & Trpc1 & AAGGCTGCTTTCCGTTCACT & TACATCTCAAGCCGCAAGCA \\
\hline \multirow{2}{*}{ Mm00435673_m1 } & Trpc3 & ATACCTTCACCATGCGGAGC & TCACTGCTTGGAGTGCTGAG \\
\hline & Trpc5 & AGCAGCACTCTATGTGGCAG & GCACCCCGGATTTCACCTAA \\
\hline \multirow{2}{*}{ Mm00479987_m1 } & Calm1a & TGTCAGCAGCCAGTTTACC & ACCCGTTTCCTGCACATCAT \\
\hline & Calm2 & AAGTGTGGAGTTGTGAGCGT & ACGAGTGAGTACCGGACAGA \\
\hline Mm01247293_m1 & Calm3 & TGCCCGTTCTCCTGATCTCT & GCGTTTGCTAGAACCGGGTA \\
\hline \multirow[t]{3}{*}{ Mm01317678_m1 } & Calm4 & GTGTTCCGGGTCTTTGACCA & САTTCAGCTCСTCСTCGGAC \\
\hline & Post & GTGCTAGCTGCGATGACTCT & GATGGTTTCAGGAAGGCCGA \\
\hline & Golli & AGCATCTGAGAAGGCCAGTAA & АTCTGCCTCCCCAAACACATC \\
\hline Mm00501184_m1 & Gapdh & TGACTCTACCCACGGCAAGTTCAA & ACGACATACTCAGCACCAGCATCA \\
\hline
\end{tabular}

Mm00448413_m1

Mm00727761_s1

Mm00477208_m1

Mm00443100_m1

Mm00501325_m1

Mm00501389_m1

Mm01175211_m1

Mm00465877_m1

Mm01328421_m1 Mm01222827_m1

Mm00771682_g1

Mm00448225_m1

Mm00490758_m1

Mm00728482_s1

Mm00486423_m1 Mm01223103_m1 Mm00441975_m1

were centrifuged at $16,000 \times \mathrm{g}$ for $30 \mathrm{~min}$ at $4^{\circ} \mathrm{C}$. After measuring the protein concentrations with the $\mathrm{BC}$ Assay Protein Quantification Kit (Interchim), $100 \mu \mathrm{g}$ protein was loaded on 3-8\% Tris-acetate gel (Invitrogen). The proteins were transferred to a nitrocellulose membrane using the iBlot system (Invitrogen), fixed with Ponceau S solution (Sigma) for $20 \mathrm{~s}$, and then blocked for $1 \mathrm{~h}$ with $3 \%$ dry non-fat milk in phosphate-buffered saline with $0.01 \%$ Tween-20 (PBS-T) at room temperature under gentle shaking. The membranes were incubated overnight at $4^{\circ} \mathrm{C}$ against actin (MAB1501, 1:4000, Millipore) and huntingtin (1756-1, 1:1000, Epitomics) diluted in PBS-T that contained 3\% dry nonfat milk. After washing three times with PBS-T, the membranes were incubated with the secondary antibody IRDye 800 goat antirabbit immunoglobulin $\mathrm{G}(\mathrm{IgG})$ and IRDye 680 goat anti-mouse IgG, (both from Licor, 926-32211 and 926-32220, respectively) for $1 \mathrm{~h}$ at room temperature. The secondary antibodies were diluted to 1:30000 in 3\% dry non-fat milk in PBS-T. Afterward, the membranes were washed three times with PBS-T and scanned for infrared fluorescence at 680 or $800 \mathrm{~nm}$ using an Odyssey scanner system (Licor). Brain tissue was homogenized on ice using a glass-homogenizer (70 strokes) and cleared by centrifugation at $12,000 \times \mathrm{g}$ for $20 \mathrm{~min}$. Protein extracts $(20 \mu \mathrm{g})$ were separated on 10\% SDS-polyacrylamide gel electrophoresis (PAGE), transferred to a Protran nitrocellulose membrane (Whatman), and blocked for $2 \mathrm{~h}$ at room temperature in TBS-T: $50 \mathrm{mM}$ Tris- $\mathrm{HCl}$, $\mathrm{pH} 7.5,150 \mathrm{mM} \mathrm{NaCl}$, and $0.1 \%$ Tween 20 plus $5 \%$ dry non-fat milk. Nitrocellulose sheets were then incubated at $4^{\circ} \mathrm{C}$ overnight in blocking solution with primary monoclonal antibodies against Mm01276999_m1 HAP1 (611302, DB Transduction Laboratories) diluted to 1:300; 
CacyBP/SIP (ab51288, Abcam), 1:1000; Cib2 (H00010518-A01, Abnova), 1:100; pan-cadherin (ab6528, Abcam), 1:2500; as well as with primary polyclonal antibodies against CIB1 (11823-1-AP, Proteintech Europe), 1:100; CALB2 (7699/3H, Swant), 1:500; and glyceraldehyde 3-phosphate dehydrogenase (sc-25778, GAPDH) (Santa Cruz), 1:1000. The appropriate horseradish peroxidaseconjugated secondary antibody anti-mouse IgG (A9044) and anti-rabbit IgG (A0545), both from Sigma, was added at a dilution of 1:10000 for $1 \mathrm{~h}$ at room temperature. The peroxidase was detected with enhanced chemiluminescence (Amersham Biosciences). The intensity of the bands was measured using an ImageQuant LAS 4000 (GE Healthcare) and Quantity One software (Bio-Rad). Densitometry was then performed using the intensity of GAPDH or pan-cadherin bands as an internal standard depending on the molecular weight of the analyzed protein.

\section{STATISTICAL ANALYSIS}

To calculate $p$-values for differences in gene expression levels between the same brain regions (striatum or motor cortex or cerebellum) in wildtype and transgenic mice, we applied two-tailed paired Student's $t$-test using $\Delta \mathrm{CT}$ values for the results obtained with the RT-qPCR arrays (Figure 1; $n=3$ ) or relative values for the results obtained with individual qRT-PCRs (Table 4; $n=3$ ). The degree of significance $v s$. control is indicated by asterisks: ${ }^{*} p<0.05,{ }^{* *} p<0.005,{ }^{* * *} p<0.0005$ (ns, not significant, $p>$ $0.05)$. To make the criteria for statistical significance more stringent, the Bonferroni correction $(p<0.00052)$ was applied. This was calculated as the $p$-value $(0.05)$ divided by 96 , which is the number of studied genes (Noble, 2009).

\section{RESULTS}

\section{DIFFERENCES IN GENE EXPRESSION BETWEEN TRANSGENIC HD MICE (YAC128) AND WILDTYPE MICE IN THE STRIATUM, CEREBELLUM AND MOTOR CORTEX}

To estimate the relative mRNA levels of the ensemble of genes that encode the calciosome, custom-made RT-qPCR arrays were prepared containing genes expressed in the mouse brain according to the Allen Brain Atlas (www.brain-map.org; accessed July 2, 2013; Table 1). We dissected the striatum from 3-month-old YAC128 mice and non-transgenic controls and analyzed the expression of four standards, 57 calciosome genes, as well as genes implicated in HD and Alzheimer's disease (AD) using two technical replicates. For comparison, we also performed these analyses using preparations of the cerebellum and motor cortex.

All 96 genes present in our custom-made PCR arrays were detected in the forebrain. In the striatum, $\sim 31$ of the analyzed genes exhibited a statistically significant difference $(p<0.05)$ in expression between YAC128 mice and control mice (Figure 1A). Twenty-seven genes were expressed at a higher level in HD transgenic mice compared with non-transgenic mice, and four genes were expressed at a lower level. Statistically significant differences between gene expression in YAC128 mice and control mice were observed in the cerebellum and motor cortex. In the cerebellum, eight genes exhibited enhanced expression in HD transgenic mice, and in the cortex, two had enhanced expression and eight reduced expression relative to control animals (Figures 1B,C).

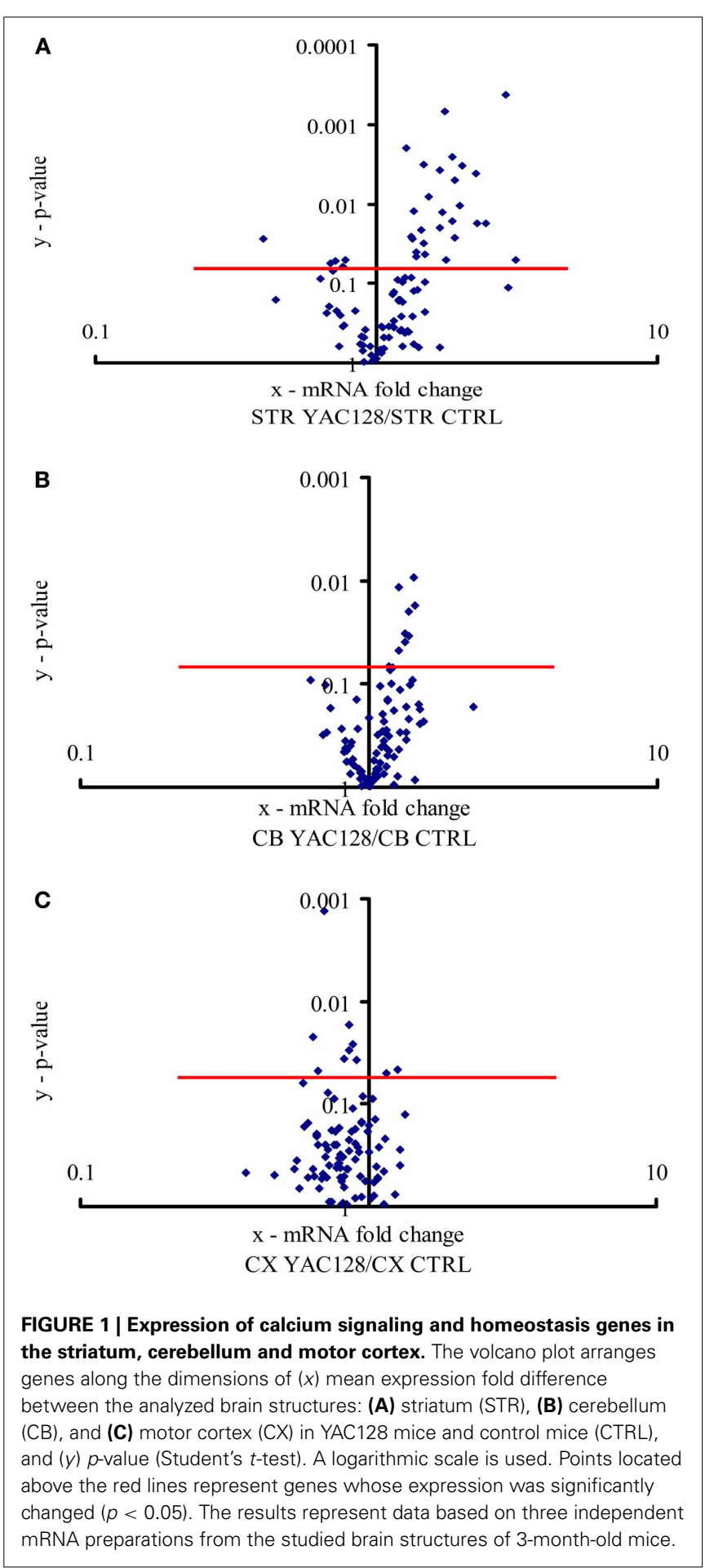

\section{EXPRESSION OF GENES POTENTIALLY IMPLICATED IN HD IN YAC128 MICE}

To identify genes with potential significance in this HD animal model, we focused on genes whose expression was changed at least 1.5-fold in the striatum; namely, Hap1 (huntingtinassociated protein 1), Calb2 (calretinin), Aph1 (anterior pharynx defective 1 homolog) isoforms a, b and c, Psenen (presenilin 
enhancer 2 homolog), Psen2 (presenilin 2), Cib1 (calmyrin 1), CacyBP/SIP (calcyclin-binding protein), Calr (calreticulin), and Cib2 (calmyrin 2).

The expression of Hap1, Calb2, and Aph1b was approximately three-times higher in the striatum of HD mice compared to control mice. When we employed the Bonferroni correction, of the 31 genes whose mRNA levels were significantly changed in the striatum in YAC128 mice, only the Aph $1 b$ gene met the criterion of $p<0.00052$.

The most prominent changes in gene expression between 3-month-old YAC128 mice and non-transgenic mice were observed in the striatum (Figure 1A). This is consistent with the fact that the first changes that occur in the brains of patients suffering from HD also appear in the striatum (Vonsattel et al., 1985). Since the clinical and pathological changes become more apparent with age, we analyzed the same brain structure in 6month-old YAC128 mice and wildtype animals. Equal amounts of cDNA obtained from the striatum in three transgenic HD mice and three control mice were prepared, and real-time PCR was performed using our RT-qPCR array. The most significant changes that were observed in the striatum in 3-month-old YAC128 mice were also observed in 6-month-old HD animals. The genes whose expression exhibited at least a 1.3-fold increase in 6-month-old mice were compared to the list of genes that were overexpressed in the striatum in 3-month-old mice. Eleven genes were identified that had significantly higher expression in the striatum in mice YAC128 of both ages (Table 3 ) comparing to control mice.

In addition to the genes that had higher expression in the striatum in YAC128 mice, two genes were downregulated in both age cohorts: Bace1, a gene that encodes the $\beta$-secretase component ( $\beta$-site APP cleaving enzyme 1 ), and $R g s 4$, a member of the family of guanosine triphosphatase (GTPase)-activating proteins that regulate $\mathrm{G}_{q}$ and $\mathrm{G}_{i}$ proteins (Huang et al., 1997). However, in contrast to the up to three-fold increases associated with the other genes, the level of these two mRNAs decreased by only $10-30 \%$ (0.7-0.9-fold).

In the group of genes presented in Table 3, only Aph $1 b$ had $p<$ 0.00052 , as calculated using the Bonferroni correction. However, we also identified 12 other genes that did not meet the Bonferroni correction criterion. Nevertheless, for these genes, we observed high relative quantification and the same extent of mRNA changes in the striatum in both HD mice groups compared with control mice. We therefore chose to check some of these results at the protein level.

\section{VERIFICATION OF GENE EXPRESSION DATA AT THE PROTEIN LEVEL DETERMINED BY WESTERN BLOT}

Protein extracts from the striatum of 3-, 4-, and 6-month-old YAC128 and age-matched control mice were prepared, separated by SDS-PAGE, and incubated with primary antibodies against HAP1, CacyBP/SIP, CALB2, CIB2, CIB1 and GAPDH or pancadherin as loading controls. In six independent western blot experiments, we observed increase in CacyBP/SIP and CIB2 proteins in the striatum of YAC128 mice compared to control mice (Figures 2B,D). In five out of six blots there was an increase in HAP1 protein (Figure 2A) and a decrease in CIB1 (Figure 2E). In four out of six blots we observed increase in CALB2 (Figure 2C).
This confirms that screening for changes at the mRNA level is feasible but must be followed by verification at the protein level.

\section{REDUCTION OF SOCE BY EXPRESSION OF MUTATED HUNTINGTIN IN PC12 CELLS}

To functionally evaluate our results at the cellular level, we adopted a cellular model of HD; namely, inducible PC12 cells (Apostol et al., 2003), in which an expanded HTT with 73 glutamines is expressed upon treatment with the insect steroid ponasterone A (Figure 3A). We first investigated the effects of mutated HTT on intracellular $\mathrm{Ca}^{2+}$ homeostasis and the expression of the genes identified in the first part of the study. Cultured PC12 cells were separated into two portions; one was treated with ponasterone A for $48 \mathrm{~h}$ to induce the expression of mutated human HTT and the other one with vehicle. For the calcium imaging experiments, both cultures were incubated with Fura2AM. SOCE was examined by incubating the cells in a calciumfree medium ( $0.5 \mathrm{mM}$ EGTA) followed by store depletion with $2 \mu \mathrm{M}$ of the SERCA pump inhibitor thapsigargin (TG). The reintroduction of extracellular $\mathrm{Ca}^{2+}$ evoked a robust $\mathrm{Ca}^{2+}$ influx that corresponded to SOCE in both types of PC12 cells. However, cells with human HTT exhibited a significant reduction in $\mathrm{Ca}^{2+}$ reentry compared with control cells (Figure 3C).

In order to check if mutant HTT affect the $\mathrm{ER} \mathrm{Ca}^{2+}$ store content in PC12 cells, we incubated cells either in the absence of extracellular $\mathrm{Ca}^{2+}$ or in the presence of $2 \mathrm{mM} \mathrm{Ca}^{2+}$. Next, $2 \mu \mathrm{M}$ TG was added to inhibit $\mathrm{Ca}^{2+}$-ATPase and the amount of calcium ions released from ER were detected. The TG-releasable pool was smaller under both conditions in the cells with induced expression of HTT, but the difference did not reach statistical significance (Figures 3D,E). PC12 cells expressing mutant HTT exhibited significant differences in SOCE, which is in line with our hypothesis that HD is caused or accompanied by changes in the calciosome.

Next, we tried to verify the expression of those calciosome components that were regulated in YAC128 mice using induced PC12 cells. Surprisingly, we did not detect changes in Hap1, Calb2, Aph1b, or CacyBP/SIP gene expression in this model. We then chose genes that encode proteins known to be directly involved in SOCE or genes that encoded proteins that regulate SOCE activity to find a possible explanation for the reduced SOCE in inducible huntingtin-expressing PC12 cells. As shown in Table 4, we detected a statistically significant downregulation of Orai calcium release-activated calcium modulator 2 (Orai2), calmodulin 3 (Calm3), and septin 4 (Sept4). No changes in other genes that encode proteins that directly participate in SOCE, such as STIMs, RYRs, $\mathrm{IP}_{3} \mathrm{Rs}$, SERCAs, and other Orais, were detected. No changes were found in other genes that encode proteins that indirectly regulate SOCE, such as TRPCs, CALMs, Golli, Post, and TMEM66. These data suggest that different mechanisms contribute to changes in calcium homeostasis in inducible huntingtin-expressing PC12 cells and transgenic YAC128 mice.

\section{DISCUSSION}

Several studies have investigated gene expression in HD models and HD patients using microarray analysis and discovered genes whose expression is altered (Luthi-Carter et al., 2000, 2002a,b; 
Table 3 | Gene expression analysis in the striatum in YAC128 mice.

\begin{tabular}{|c|c|c|c|c|}
\hline \multirow[t]{2}{*}{ Gene symbol } & \multirow[t]{2}{*}{ Gene name } & \multicolumn{2}{|c|}{ Striatum (3 months) } & \multirow{2}{*}{$\begin{array}{l}\text { Striatum (6 months) } \\
\text { Relative quantification }\end{array}$} \\
\hline & & Student's $t$-test & Relative quantification & \\
\hline Hap1 & huntingtin-associated protein 1 & * & 3.1 & 1.8 \\
\hline Calb2 & calbindin 2 (calretinin) & ns & 2.9 & 2.1 \\
\hline Aph $1 b$ & anterior pharynx defective $1 \mathrm{~b}$ homolog (C. elegans) & $* * *$ & 2.9 & 1.9 \\
\hline Psenen & presenilin enhancer 2 homolog (C. elegans) & * & 2.3 & 1.5 \\
\hline Aph1a & anterior pharynx defective 1a homolog (C. elegans) & ** & 2.3 & 1.9 \\
\hline Psen2 & presenilin 2 & * & 2.0 & 1.6 \\
\hline Cib1 & calcium and integrin binding 1 (calmyrin 1) & ** & 2.0 & 1.3 \\
\hline CacyBP/SIP & calcyclin binding protein & * & 1.8 & 1.6 \\
\hline Aph1c & anterior pharynx defective $1 \mathrm{c}$ homolog (C. elegans) & * & 1.7 & 1.9 \\
\hline Calr & calreticulin & * & 1.5 & 1.6 \\
\hline Cib2 & calcium and integrin binding family member 2 & ** & 1.5 & 1.8 \\
\hline Bace1 & beta-site APP cleaving enzyme 1 & * & 0.7 & 0.8 \\
\hline Rgs4 & regulator of G-protein signaling 4 & * & 0.8 & 0.9 \\
\hline
\end{tabular}

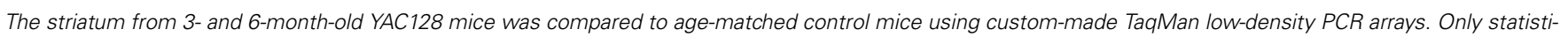

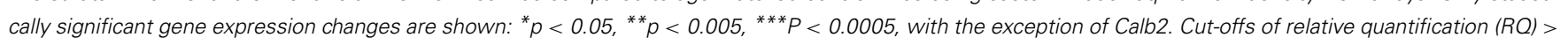

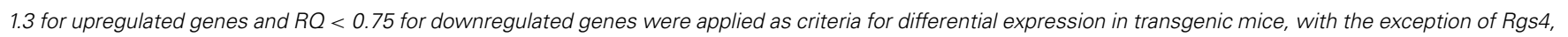

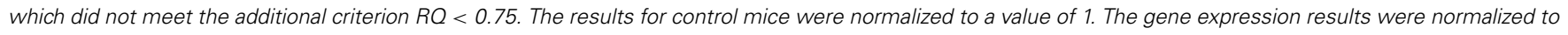

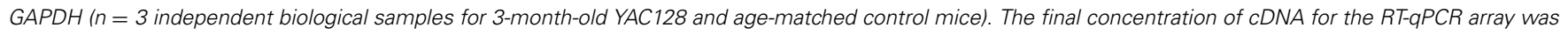

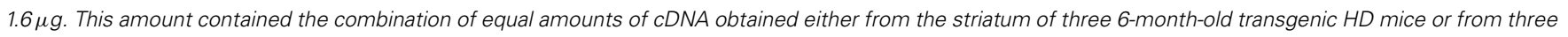
age-matched control mice.

\begin{tabular}{llll}
\hline $\begin{array}{l}\text { Table } \mathbf{4} \text { | } \\
\text { cells. }\end{array}$ & & \\
\hline $\begin{array}{l}\text { Gene } \\
\text { symbol }\end{array}$ & Gene name & Student's $\boldsymbol{t}$-test & $\begin{array}{l}\text { Relative } \\
\text { quantification }\end{array}$ \\
\hline Orai2 & Orai calcium & & 0.8 \\
& $\begin{array}{l}\text { release-activated } \\
\text { calcium } \\
\text { modulator 2 }\end{array}$ & $*$ & \\
calm3 & calmodulin 3 & $*$ & 0.8 \\
Sept4 & septin 4 & $*$ & 0.8 \\
\hline
\end{tabular}

PC12 cells induced by ponasterone A were compared to uninduced cells using qRT-PCR. The gene expression results were normalized to GAPDH. Data from uninduced PC12 cells were normalized to a value of 1. Only statistically significant gene expression changes are shown $\left(^{*} p<0.05\right)$. The results represent data based on three independent mRNA preparations.

Desplats et al., 2006; Hodges et al., 2006). We expanded these studies to the YAC128 transgenic mice because they exhibit a slow, long-term progression of symptoms characteristic of HD (Slow et al., 2003), which may help identify the primary effects of mutant HTT. We focused on genes that encode members of calcium signalosomes (calciosome) because changes in calcium signaling and homeostasis components have been suggested to be early steps in the development of HD (Giacomello et al., 2011, 2013).

We found that $\sim 32 \%$ of the analyzed genes in the striatum exhibited statistically significant changes in expression in YAC128 mice compared with control mice (Figure 1A). In the cerebellum and motor cortex, the levels of expression of fewer genes differed between YAC128 mice and wild type mice (Figures 1B,C), which is consistent with changes occurring first in the striatum of patients suffering from HD (Vonsattel et al., 1985). In the striatum, one gene, $A p h 1 b$, was positively regulated, even after a Bonferroni correction $(p<0.00052)$ to correct for multiple testing. Nevertheless, $\sim 13 \%$ of genes in the striatum met the arbitrary criteria after cut-offs of relative quantification results (RQ $>1.3$ for upregulated genes and $\mathrm{RQ}<0.75$ for downregulated genes) were applied (Table 3 ). They were characterized by the same extent of mRNA changes in the striatum in both groups, 3- and 6-month-old HD mice, as compared with control mice. Western blots were performed to check, if the change of specific mRNA level affect the level of a particular protein (Figure 2).

At the top of our list, presenting the highest score for relative quantification in the striatum in transgenic YAC128 mice compared with control mice, is the mRNA coding for huntingtin-associated protein 1 (Table 3). Our results revealed a three-fold increase in the expression of Hapl in the striatum in YAC128 mice compared with control mice. This is consistent with data obtained using another transgenic mouse model, R6/2. The enrichment of several mRNAs, including Hap1 (although not statistically significant), was observed in neuronal nitric-oxide-synthase-positive interneurons compared with medium spiny projection neurons (Zucker et al., 2005).

Hap1 did not meet the criterion of the Bonferroni correction $(p<0.00052)$, however, an increase in HAP1 protein was observed in brain extracts from five out of six studied YAC128 mice using western blotting (Figure 2A). In these five samples about 1.8-2.8-fold increase at the protein level of HAP1 was observed, except one sample, which exhibited 0.46-fold decrease of HAP1. Also, this sample showed unusually high level of CIB2 protein as compared to other five samples (Figure 2D). HAP1 is known to play a role in signal transduction, the regulation 


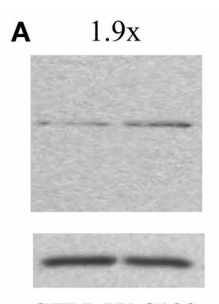

CTRL YAC128

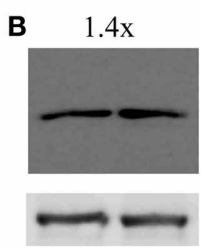

CTRL YAC128

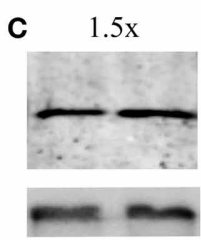

CTRL YAC128

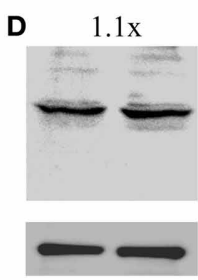

CTRL YAC128

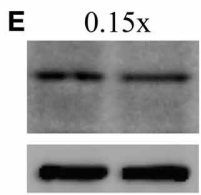

CTRL YAC128

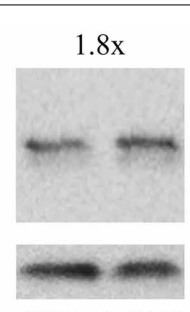

CTRL YAC128

$1.2 \mathrm{x}$

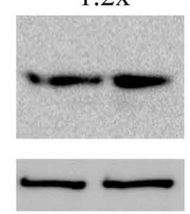

CTRL YAC128

$2.50 x$

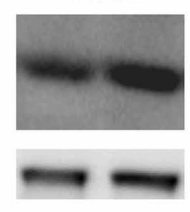

CTRL YAC128

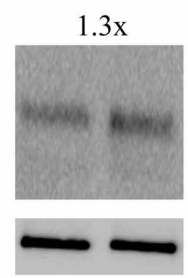

CTRL YAC128

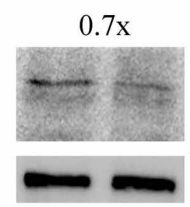

CTRL YAC128

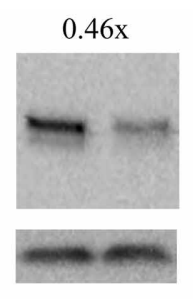

CTRL YAC128

$1.5 \mathrm{x}$

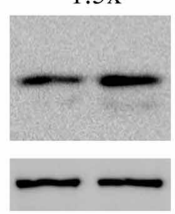

CTRL YAC128

$0.79 x$

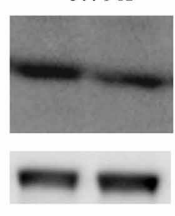

CTRL YAC128

$4.6 x$

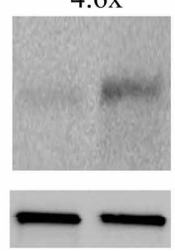

CTRL YAC128

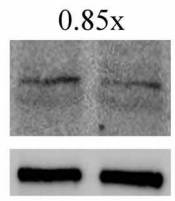

CTRL YAC128

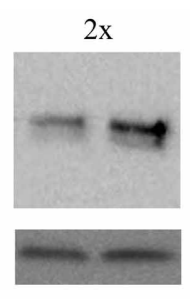

CTRL YAC128

$2.1 \mathrm{x}$

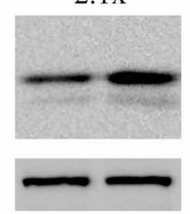

CTRL YAC128

$2.25 x$

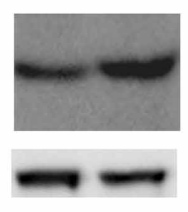

CTRL YAC128

$1.3 \mathrm{x}$

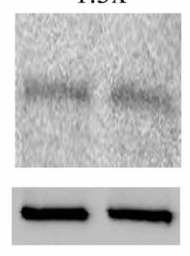

CTRL YAC128

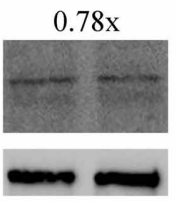

CTRL YAC128

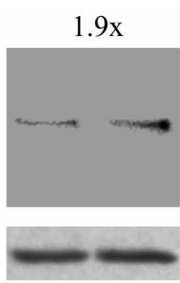

CTRL YAC 128

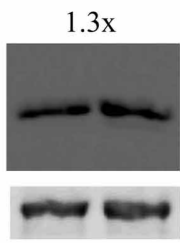

CTRL YAC128

$1.3 \mathrm{x}$

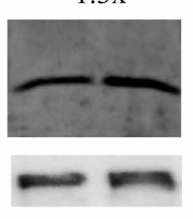

CTRL YAC128

$1.6 \mathrm{x}$

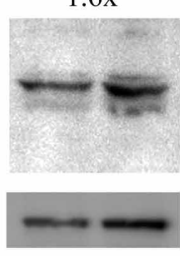

CTRL YAC128

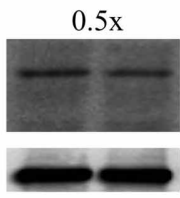

CTRL YAC 128

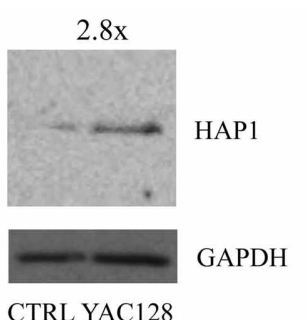

CTRL YAC128

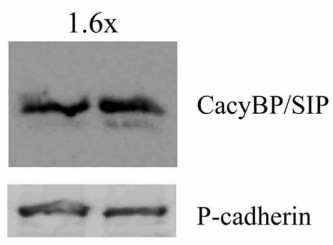

CTRL YAC128

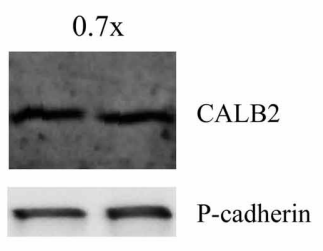

CTRL YAC128
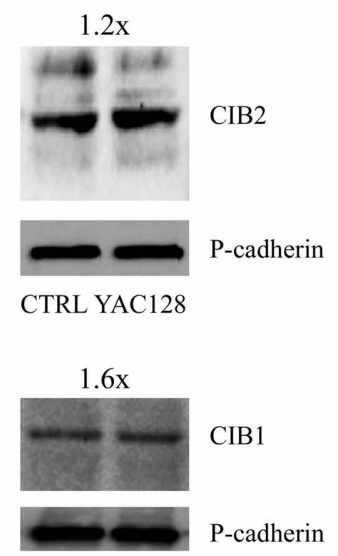

CTRL YAC128
FIGURE 2 | Protein expression analysis in the striatum in YAC128 mice. Immunoblots of HAP1 (A), CacyBP/SIP (B), CALB2 (C), CIB2 (D), and CIB1 (E) in the striatum of $Y A C 128$ mice and age-matched control mice (CTRL) are shown. First two blots show the data from 3- month-old mice, third and fourth blots-from 4-month-old mice, and fifth and sixth blots from 6-month-old animals. $20 \mu \mathrm{g}$ of protein was loaded on the gel. HAP1 densitometry was performed using the intensity of GAPDH (A). Pan-cadherin bands were used as an internal standard for CacyBP/SIP (B), CALB2 (C), CIB2 (D), and CIB1 (E). The fold change of the studied proteins is shown above the immunoblots. of vesicular transport, gene transcription, and the regulation of membrane receptor recycling ( $\mathrm{Wu}$ and $\mathrm{Zhou,} \mathrm{2009).} \mathrm{It} \mathrm{was} \mathrm{iden-}$ tified as an HTT-interacting protein in yeast two-hybrid screens (Li et al., 1995). HAP1 protein binds more tightly to HTT with an expanded glutamine repeat than to wildtype HTT, and the binding is enhanced by lengthening the glutamine repeat ( $\mathrm{Li}$ et al., 1998). A IP ${ }_{3}$ R1-HAP1A-HTT ternary complex was identified in the brain that facilitates $\mathrm{IP}_{3} \mathrm{R} 1$-mediated intracellular $\mathrm{Ca}^{2+}$ release in MSNs and is activated only by mutant HTT (Tang et al., 2003). Interactions between HTT and the $\mathrm{IP}_{3} \mathrm{R} 1 \mathrm{C}$-terminus depend on both, the presence of HAP1 and polyglutamine expansion. Mutant HTT can bind to the $\mathrm{IP}_{3} \mathrm{R} 1 \mathrm{C}$-terminus either directly or indirectly through HAP1. The functional effects of mutant HTT on $\mathrm{IP}_{3} \mathrm{R} 1$-mediated $\mathrm{Ca}^{2+}$ release are attenuated in medium spiny striatal neurons in Hapl knockout mice compared with MSNs in wildtype mice (Tang et al., 2004).

Members of calcium signalosomes, which were significantly upregulated in the striatum in YAC128 mice, were Calb2, CacyBP/SIP, Cib1, Cib2, and Carl. An increase at the protein level in samples from six animals was observed for CacyBP/SIP protein 


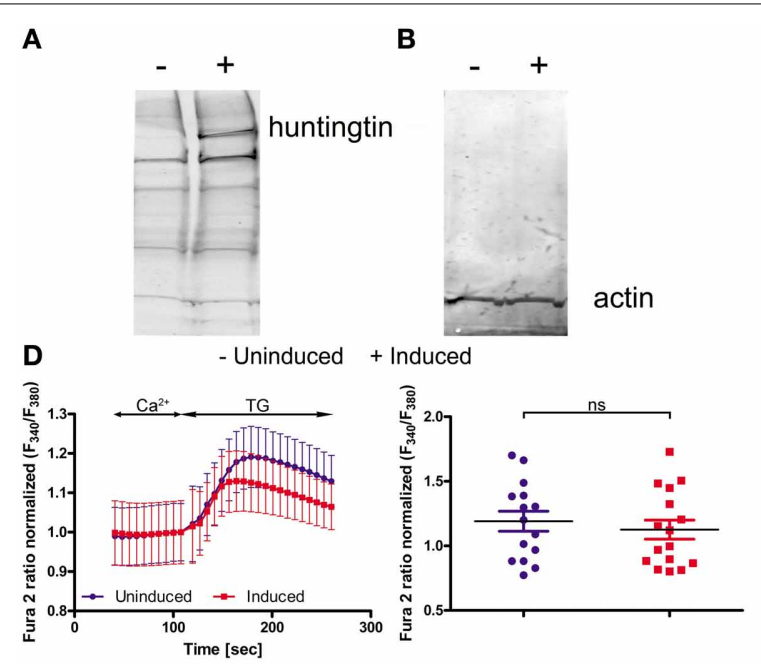

FIGURE 3 | Huntingtin expression in PC12 cells reduces SOCE. Immunoblots of huntingtin (A) and actin (B) in induced (+) and uninduced (-) PC12 cells are shown. Ratiometric Fura-2 analysis of uninduced and induced PC12 cells was performed on a BD Pathway high-content imaging system. (C) SOCE measurements began in a buffer supplemented with $0.5 \mathrm{mM}$ EGTA, which was then replaced by a buffer with $0.5 \mathrm{mM}$ EGTA and $2 \mu \mathrm{M}$ thapsigargin (TG). After $2.5 \mathrm{~min}$, the readdition of $2 \mathrm{mM} \mathrm{Ca}^{2+}$ to the extracellular media resulted in $\mathrm{Ca}^{2+}$ influx. The traces show only $\mathrm{Ca}^{2+}$ readdition after store depletion. $\mathrm{F}_{340} / \mathrm{F}_{380}$ values beginning just before the readdition of $\mathrm{Ca}^{2+}$ were normalized to the same values (1). The ER calcium stores were depleted
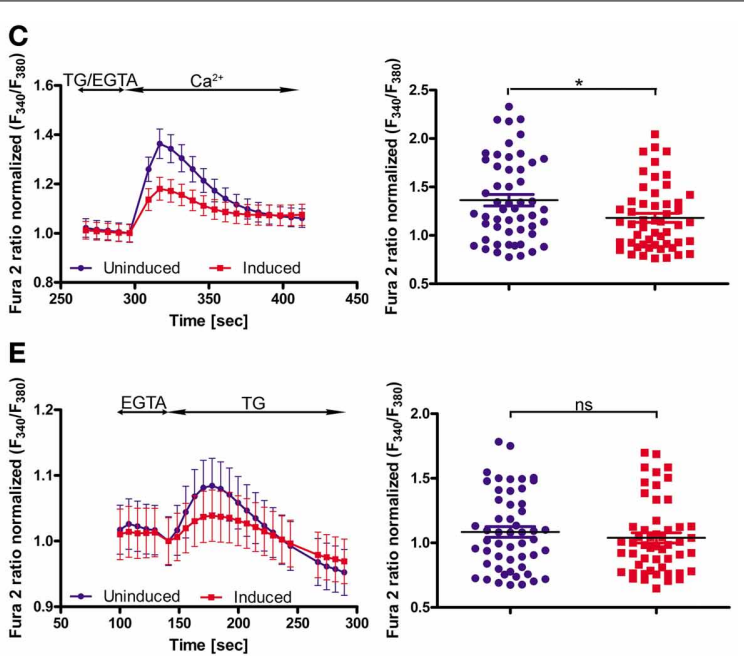

by the addition of $2 \mu \mathrm{M}$ TG in the presence (D) or absence (E) of extracellular $\mathrm{Ca}^{2+}$. $\mathrm{F}_{340} / \mathrm{F}_{380}$ values beginning just before the addition of TG were normalized to the same values (1) (C-E, left panels). The data from different experiments were averaged (C-E, right panels). Summary data present the maximum (peak) of the $F_{340} / F_{380}$ ratio after the addition of $\mathrm{Ca}^{2+}$ or $\mathrm{TG}$ are expressed as the mean $\pm \mathrm{SD}$ of the data shown in the left panels. ${ }^{*} p<0.05$; ns, not significant $(p>0.05)$. Averaged traces from (C) 52 individually measured wells that contained a total of $\sim 2,000$ cells per trace, (D) 52 individually measured wells that contained a total of $\sim 2,000$ cells per trace, and (E) 16 individually measured wells that contained a total of $\sim 500$ cells per trace. and CIB2 (Figures 2B,D). CALB2 protein increased in four samples $(1.5,2.5,2.25$, and 1.3-fold), and two were characterized by a slightly lower expression of CALB2 in the striatum $(0.79$ and 0.7 -fold). Taking into account the possible fluctuations in neuronal calcium, this result can be explained by the fact that some antibodies to calcium-binding proteins, including CALB2, preferentially recognize particular calcium-induced protein conformations (Winsky and Kuznicki, 1996). CALB2 is a member of the EF-hand family of calcium-binding proteins, which interacts with wildtype HTT but preferentially with mutant HTT (Dong et al., 2012). In neuronal cell models of HD, the overexpression of CALB2 reduced mutant HTT-induced cytotoxicity, whereas knockdown of Calb2 enhanced mutant HTT-induced neuronal cell death (Dong et al., 2012). It was suggested that CALB2 may protect only medium-sized neurons against neurodegeneration in HD (Cicchetti and Parent, 1996). However, neurodegenerative processes that play a role in HD led to a decrease in the density of CALB2 in large striatal interneurons, without causing their death (Massouh et al., 2008). An increase in the expression of Calb2 in the striatum in YAC128 mice may indicate the presence of a defense mechanism against mutant HTT-induced cytotoxicity. However, high-throughput analysis did not identify an increase in Calb2 in HD patients (Hodges et al., 2006). In contrast, a decrease in the expression of Calb1, which encodes another 6 EF-hands calcium binding protein, calbindin, was found in postmortem brain samples from the caudate of HD patients (Hodges et al., 2006). This is consistent with the substantial loss of neurons in the neostriatum that contain calbindin, suggesting that a failure of calcium buffering may contribute to cell death in HD (Seto-Ohshima et al., 1988).

The expression of CacyBP/SIP was also significantly upregulated in the striatum in YAC128 mice relative to control mice. This result was confirmed by western blotting, which showed an increase in a $30-\mathrm{kDa}$ calcyclin (S100A6)-binding protein in all six studied samples. The CacyBP/SIP protein may play a role in the organization of microtubules and in calcium-dependent ubiquitination of target proteins (Filipek and Kuznicki, 1998; Matsuzawa and Reed, 2001; Filipek et al., 2002, 2008; Jurewicz et al., 2013).

The statistically significant upregulation of Cib1 mRNA in YAC128 mice is consistent with a seven-fold increase in this gene's mRNA in microarray studies of the striatum in HD patients (Hodges et al., 2006). However, at the protein level this cannot be confirmed (Figure 2E), since we observed downregulation of CIB1 in samples from the striatum of five animals $(0.15,0.7,0.85$, 0.78 , and 0.5 -fold) and an increase in one (1.6-fold). We detected a statistically significant increase in Cib2 mRNA and this result was confirmed by six independent western blotting experiments, showing an increase in CIB2 protein (Figure 2D). CIBs are EFhand $\mathrm{Ca}^{2+}$ binding proteins identified in the brain (Bernstein et al., 2005; Blazejczyk et al., 2009). CIB1 has been implicated in neurodegenerative processes in $\mathrm{AD}$, in which it was shown to interact with presenilin 2, but not presenilin 1 (Blazejczyk et al., 2006), but the role of CIBs in HD has not yet been confirmed.

Another gene that was significantly upregulated on the mRNA level in the striatum of YAC128 mice compared with control mice 
was Calr, however, the microarray studies did not detect upregulation of Calr in brain structures in HD patients (Hodges et al., 2006). Calreticulin is a multifunctional protein that acts as a transcription factor in the nucleus or binds $\mathrm{Ca}^{2+}$ in the ER (Michalak et al., 2009).

We detected statistically significant changes in the gene expression of Psen 2 mRNA in YAC128 mice and other components of $\gamma$-secretase complex, such as Psenen and Aph1 isoforms. Considering that only the $A p h 1 b$ gene met the criterion for Bonferroni correction, we speculate that this gene may by involved in the pathogenesis of HD. APH1 encoded by the Aph1 gene is required for proteolytic activity and binds to the $\gamma$-secretase complex (Lee et al., 2004). Presenilins and Psenen are involved in the cleavage of the Notch receptor and APP processing (Francis et al., 2002; Goutte et al., 2002; Luo et al., 2003). Moreover, PSENs participate also in the modulation of $\mathrm{Ca}^{2+}$ signaling in the ER (Leissring et al., 2000; Supnet and Bezprozvanny, 2011). Thus, one can associate the altered gene expression of $\gamma$-secretase components with abnormal proteolytic activity in HD (Tarlac and Storey, 2003). It was suggested that HTT-interacting protein HIP1 (huntingtin-interacting protein 1) may provide a functional link between non-canonical Notch signaling-mediated neurogenesis through a deltex-dependent pathway (Moores et al., 2008).

In our experiments, most of the genes exhibited upregulation, in contrast to the results obtained from Affymetrix oligonucleotide array studies in HD R6/2 mice (Desplats et al., 2006), in which a decrease in the expression level of a majority of the genes was observed. R6/2 mice express exon 1 of human HD gene carrying 116 CAG repeats and exhibit a more progressive neurological phenotype (Mangiarini et al., 1996). However, comparing the results of high-throughput studies with our present study, which focused on a small group of genes, we identified some similarities. We and Desplats et al. (2006) detected a decrease in the expression of the $R g s 4$ gene that encodes a protein involved in the regulation of G-protein signaling. Similarly, statistically significant downregulation of $R g s 4$ was found in the striatum and motor cortex in HD patients using Affymetrix GeneChip microarrays (Hodges et al., 2006). The RGS4 protein inhibits $\mathrm{G}_{q}$, which, in turn, attenuates intracellular calcium signaling via phospholipase C (Berman et al., 1996). This is in line with results suggesting a cytosolic and mitochondrial $\mathrm{Ca}^{2+}$ overload in $\mathrm{HD}$ (Bezprozvanny and Hayden, 2004).

Cellular models of HD are another tool to detect the early, direct effects of mutant HTT without the influence of other secondary processes in advanced stages of the disease. We used PC12 cells with inducible expression of mutant HTT. Others have characterized this model in regard to HTT aggregates: 1 day after induction, only a few cells that expressed mutant HTT contained aggregates, whereas 5 days after induction, nearly all of the cells contained visible aggregates (Cong et al., 2005). We analyzed the cells 2 days after induction of HTT expression, which exhibited overexpression of expanded HTT protein (Figure 3A) and unchanged level of actin protein (Figure 3B) as found by immunoblotting. We observed a statistically significant reduction of calcium influx during SOCE, but not calcium content in the $\mathrm{ER}$, in inducible huntingtin-expressing PC12 cells compared with control uninduced cells. This indicates that despite the likely association of mutant HTT with the ER (Rockabrand et al., 2007), the $\mathrm{Ca}^{2+}$ content of PC12 cells is not changed. Reduced activity of the SOCE in induced PC12 cells might be explained by our finding that Orai2, Sept4 and Calm3 are downregulated. The Orai calcium channel is activated during SOCE (Frischauf et al., 2008). Septin 4 has been shown to facilitate interactions between STIM1 and Orail proteins (Sharma et al., 2013). Microarray studies of the caudate from HD patients detected a seven-fold decrease in the expression of Calm3 (Hodges et al., 2006). In induced PC12 cells, a decrease in the expression of another isoform of calmodulin, Calm1, has been described (van Roon-Mom et al., 2008). Calmodulins are multifunctional intermediate messenger proteins that transduce calcium signals by binding calcium ions and then modifying their interactions with various target proteins (Chin and Means, 2000). A previous study found that HTT interacts with calmodulin and that the expansion of polyglutamine altered this interaction (Bao et al., 1996).

In contrast to the observed decrease in SOCE in PC12 cells (this work) in cultured MSNs from YAC128 mice, an increase in calcium influx during SOCE was reported by Wu and coworkers. They demonstrated a key role of TRPC1 channels in supporting the SOC pathway in HD neurons (Wu et al., 2011). However, we did not find any differences in $\operatorname{Trpc1}$ gene expression in induced PC12 cells. The differences in SOCE dysregulation observed between the YAC128 mouse model by Wu et al. and our observation in PC12 cells may result from the length of mutated HTT, which can selectively affect gene expression. Alternatively, the difference could be due to the length of time mutated HTT was expressed. MSNs from YAC128 mice used by Wu et al. were cultured from 10-14 days, while PC12 cells were analyzed 2 days after HTT induction.

Although inducible PC12 cells and transgenic YAC128 mice show different changes in calcium influx during SOCE, each of these models has characteristic features of HD, such as the deposition of HTT aggregates mainly in the nuclei of inducible PC12 cells (van Roon-Mom et al., 2008) and nuclei of MSNs in the striatum in YAC128 mice (Van Raamsdonk et al., 2007). In both models, some similarities with the results obtained from samples from HD patients as well as other HD transgenic and cellular models were observed. In our experiments, the expression of Rgs4, Hap1, Cib1, and Calm3 changed in the same direction as the changes reported by other studies.

This work presents for the first time convincing data demonstrating an increased expression of huntingtin-associated protein 1 in the striatum of HD mouse model at the mRNA and protein level. Our data showing upregulation of HAP1 are in line with those of Wu et al. (2011) when the observations of Tang et al. are taken into account (Tang et al., 2003, 2004). They found that mutant $\mathrm{Htt}$ via the interaction with $\mathrm{HAP} 1$ can activate $\mathrm{IP}_{3} \mathrm{R} 1$ in vivo. Thus, activation of SOCE reported by Wu et al. (2011) could be explained by the increased release of $\mathrm{Ca}^{2+}$ from ER due to the facilitated opening of the $\mathrm{IP}_{3} \mathrm{R} 1$. Since the dysregulation of neuronal $\mathrm{Ca}^{2+}$ represents an early event in the pathogenesis of models of HD (Zeron et al., 2002; Tang et al., 2003), the HAP1 protein might be considered as a potential therapeutic target in HD. 


\section{ACKNOWLEDGMENTS}

This study was supported by The National Centre for Research and Development in Poland in the framework of the project titled "Pathways of Store-Operated Calcium Entry (SOCE) as a novel therapeutic target in neurodegenerative diseases" (ERA.Net RUS project no NCBR/ERA NET RUS/03/2012). We thank Darragh O'Neill for excellent proofreading of the manuscript.

\section{REFERENCES}

Apostol, B. L., Kazantsev, A., Raffioni, S., Illes, K., Pallos, J., Bodai, L., et al. (2003). A cell-based assay for aggregation inhibitors as therapeutics of polyglutaminerepeat disease and validation in Drosophila. Proc. Natl. Acad. Sci. U.S.A. 100, 5950-5955. doi: 10.1073/pnas.2628045100

Bao, J., Sharp, A. H., Wagster, M. V., Becher, M., Schilling, G., Ross, C. A., et al. (1996). Expansion of polyglutamine repeat in huntingtin leads to abnormal protein interactions involving calmodulin. Proc. Natl. Acad. Sci. U.S.A. 93, 5037-5042. doi: 10.1073/pnas.93.10.5037

Berman, D. M., Kozasa, T., and Gilman, A. G. (1996). The GTPase-activating protein RGS4 stabilizes the transition state for nucleotide hydrolysis. J. Biol. Chem. 271, 27209-27212. doi: 10.1074/jbc.271.44.27209

Bernstein, H. G., Blazejczyk, M., Rudka, T., Gundelfinger, E. D., Dobrowolny, H., Bogerts, B., et al. (2005). The Alzheimer disease-related calcium-binding protein Calmyrin is present in human forebrain with an altered distribution in Alzheimer's as compared to normal ageing brains. Neuropathol. Appl. Neurobiol. 31, 314-324. doi: 10.1111/j.1365-2990.2005.00646.x

Berridge, M. J. (2012). Calcium signalling remodelling and disease. Biochem. Soc. Trans. 40, 297-309. doi: 10.1042/BST20110766

Berridge, M. J., Bootman, M. D., and Roderick, H. L. (2003). Calcium signalling: dynamics, homeostasis and remodelling. Nat. Rev. Mol. Cell Biol. 4, 517-529. doi: $10.1038 / \mathrm{nrm} 1155$

Bezprozvanny, I., and Hayden, M. R. (2004). Deranged neuronal calcium signaling and Huntington disease. Biochem. Biophys. Res. Commun. 322, 1310-1317. doi: 10.1016/j.bbrc.2004.08.035

Bezprozvanny, I. B. (2010). Calcium signaling and neurodegeneration. Acta Nat. 2, 72-82.

Billing-Marczak, K., and Kuznicki, J. (1999). Calretinin-sensor or bufferfunction still unclear. Pol. J. Pharmacol. 51, 173-178.

Blazejczyk, M., Sobczak, A., Debowska, K., Wisniewska, M. B., Kirilenko, A., Pikula, S., et al. (2009). Biochemical characterization and expression analysis of a novel EF-hand $\mathrm{Ca} 2+$ binding protein calmyrin2 (Cib2) in brain indicates its function in NMDA receptor mediated Ca2+ signaling. Arch. Biochem. Biophys. 487, 66-78. doi: 10.1016/j.abb.2009.05.002

Blazejczyk, M., Wojda, U., Sobczak, A., Spilker, C., Bernstein, H. G., Gundelfinger, E. D., et al. (2006). Ca2+-independent binding and cellular expression profiles question a significant role of calmyrin in transduction of $\mathrm{Ca} 2+$-signals to Alzheimer's disease-related presenilin 2 in forebrain. Biochim. Biophys. Acta 1762, 66-72. doi: 10.1016/j.bbadis.2005.09.006

Chin, D., and Means, A. R. (2000). Calmodulin: a prototypical calcium sensor. Trends Cell Biol. 10, 322-328. doi: 10.1016/S0962-8924(00)01800-6

Cicchetti, F., and Parent, A. (1996). Striatal interneurons in Huntington's disease: selective increase in the density of calretinin-immunoreactive medium-sized neurons. Mov. Disord. 11, 619-626. doi: 10.1002/mds.870110605

Cong, S. Y., Pepers, B. A., Evert, B. O., Rubinsztein, D. C., Roos, R. A., van Ommen, G. J., et al. (2005). Mutant huntingtin represses CBP, but not p300, by binding and protein degradation. Mol. Cell. Neurosci. 30, 560-571. doi: 10.1016/j.mcn.2005.05.003

Desplats, P. A., Kass, K. E., Gilmartin, T., Stanwood, G. D., Woodward, E. L., Head, S. R., et al. (2006). Selective deficits in the expression of striatalenriched mRNAs in Huntington's disease. J. Neurochem. 96, 743-757. doi: 10.1111/j.1471-4159.2005.03588.x

Dong, G., Gross, K., Qiao, F., Ferguson, J., Callegari, E. A., Rezvani, K., et al. (2012). Calretinin interacts with huntingtin and reduces mutant huntingtincaused cytotoxicity. J. Neurochem. 123, 437-446. doi: 10.1111/j.14714159.2012.07919.x

Filipek, A., Jastrzebska, B., Nowotny, M., and Kuznicki, J. (2002). CacyBP/SIP, a calcyclin and Siah-1-interacting protein, binds EF-hand proteins of the S100 family. J. Biol. Chem. 277, 28848-28852. doi: 10.1074/jbc.M203602200
Filipek, A., and Kuznicki, J. (1998). Molecular cloning and expression of a mouse brain cDNA encoding a novel protein target of calcyclin. J. Neurochem. 70, 1793-1798. doi: 10.1046/j.1471-4159.1998.70051793.x

Filipek, A., Schneider, G., Mietelska, A., Figiel, I., and Niewiadomska, G. (2008). Age-dependent changes in neuronal distribution of CacyBP/SIP: comparison to tubulin and the tau protein. J. Neural Transm. 115, 1257-1264. doi: 10.1007/s00702-008-0062-3

Foskett, J. K., White, C., Cheung, K. H., and Mak, D. O. (2007). Inositol trisphosphate receptor Ca2+ release channels. Physiol. Rev. 87, 593-658. doi: 10.1152/physrev.00035.2006

Francis, R., McGrath, G., Zhang, J., Ruddy, D. A., Sym, M., Apfeld, J., et al. (2002). aph-1 and pen-2 are required for Notch pathway signaling, gamma-secretase cleavage of betaAPP, and presenilin protein accumulation. Dev. Cell 3, 85-97. doi: 10.1016/S1534-5807(02)00189-2

Frischauf, I., Schindl, R., Derler, I., Bergsmann, J., Fahrner, M., and Romanin, C. (2008). The STIM/Orai coupling machinery. Channels (Austin) 2, 261-268. doi: 10.4161/chan.2.4.6705

Giacomello, M., Hudec, R., and Lopreiato, R. (2011). Huntington's disease, calcium, and mitochondria. Biofactors 37, 206-218. doi: 10.1002/biof.162

Giacomello, M., Oliveros, J. C., Naranjo, J. R., and Carafoli, E. (2013). Neuronal $\mathrm{Ca}(2+)$ dyshomeostasis in Huntington disease. Prion 7, 76-84. doi: $10.4161 /$ pri.23581

Goutte, C., Tsunozaki, M., Hale, V. A., and Priess, J. R. (2002). APH-1 is a multipass membrane protein essential for the Notch signaling pathway in Caenorhabditis elegans embryos. Proc. Natl. Acad. Sci. U.S.A. 99, 775-779. doi: 10.1073/pnas.022523499

Groenendyk, J., Lynch, J., and Michalak, M. (2004). Calreticulin, Ca2+, and calcineurin - signaling from the endoplasmic reticulum. Mol. Cells 17, 383-389.

Gruszczynska-Biegala, J., and Kuznicki, J. (2013). Native STIM2 and ORAI1 proteins form a calcium-sensitive and thapsigargin-insensitive complex in cortical neurons. J. Neurochem. 126, 727-738. doi: 10.1111/jnc.12320

Gruszczynska-Biegala, J., Pomorski, P., Wisniewska, M. B., and Kuznicki, J. (2011). Differential roles for STIM1 and STIM2 in store-operated calcium entry in rat neurons. PLoS ONE 6:e19285. doi: 10.1371/journal.pone.0019285

Hamilton, S. L. (2005). Ryanodine receptors. Cell Calcium 38, 253-260. doi: 10.1016/j.ceca.2005.06.037

Henke, N., Albrecht, P., Bouchachia, I., Ryazantseva, M., Knoll, K., Lewerenz, J., et al. (2013). The plasma membrane channel ORAI1 mediates detrimental calcium influx caused by endogenous oxidative stress. Cell Death Dis. 4:e470. doi: $10.1038 /$ cddis. 2012.216

Henke, N., Albrecht, P., Pfeiffer, A., Toutzaris, D., Zanger, K., and Methner, A. (2012). Stromal interaction molecule 1 (STIM1) is involved in the regulation of mitochondrial shape and bioenergetics and plays a role in oxidative stress. J. Biol. Chem. 287, 42042-42052. doi: 10.1074/jbc.M112.417212

Hodges, A., Strand, A. D., Aragaki, A. K., Kuhn, A., Sengstag, T., Hughes, G., et al. (2006). Regional and cellular gene expression changes in human Huntington's disease brain. Hum. Mol. Genet. 15, 965-977. doi: 10.1093/hmg/ddl013

Huang, C., Hepler, J. R., Gilman, A. G., and Mumby, S. M. (1997). Attenuation of Gi- and Gq-mediated signaling by expression of RGS4 or GAIP in mammalian cells. Proc. Natl. Acad. Sci. U.S.A. 94, 6159-6163. doi: 10.1073/pnas.94.12.6159

Huang, G. N., Zeng, W., Kim, J. Y., Yuan, J. P., Han, L., Muallem, S., et al. (2006) STIM1 carboxyl-terminus activates native SOC, I(crac) and TRPC1 channels. Nat. Cell Biol. 8, 1003-1010. doi: 10.1038/ncb1454

Jaworska, A., Dzbek, J., Styczynska, M., and Kuznicki, J. (2013). Analysis of calcium homeostasis in fresh lymphocytes from patients with sporadic Alzheimer's disease or mild cognitive impairment. Biochim. Biophys. Acta 1833, 1692-1699. doi: 10.1016/j.bbamcr.2013.01.012

Jurewicz, E., Ostrowska, Z., Jozwiak, J., Redowicz, M. J., Lesniak, W., Moraczewska, J., et al. (2013). CacyBP/SIP as a novel modulator of the thin filament. Biochim. Biophys. Acta 1833, 761-766. doi: 10.1016/j.bbamcr.2012.12.010

Klejman, M. E., Gruszczynska-Biegala, J., Skibinska-Kijek, A., Wisniewska, M. B., Misztal, K., Blazejczyk, M., et al. (2009). Expression of STIM1 in brain and puncta-like co-localization of STIM1 and ORAI1 upon depletion of $\mathrm{Ca}(2+)$ store in neurons. Neurochem. Int. 54, 49-55. doi: 10.1016/j.neuint.2008.10.005

Lee, S. F., Shah, S., Yu, C., Wigley, W. C., Li, H., Lim, M., et al. (2004). A conserved GXXXG motif in APH-1 is critical for assembly and activity of the gammasecretase complex. J. Biol. Chem. 279, 4144-4152. doi: 10.1074/jbc.M309745200

Leissring, M. A., Akbari, Y., Fanger, C. M., Cahalan, M. D., Mattson, M. P., and LaFerla, F. M. (2000). Capacitative calcium entry deficits and elevated luminal 
calcium content in mutant presenilin-1 knockin mice. J. Cell Biol. 149, 793-798. doi: $10.1083 / j \mathrm{jcb} .149 .4 .793$

Li, S. H., Hosseini, S. H., Gutekunst, C. A., Hersch, S. M., Ferrante, R. J., and Li, X. J. (1998). A human HAP1 homologue. Cloning, expression, and interaction with huntingtin. J. Biol. Chem. 273, 19220-19227. doi: 10.1074/jbc.273.30.19220

Li, S. H., and Li, X. J. (2004). Huntingtin-protein interactions and the pathogenesis of Huntington's disease. Trends Genet. 20, 146-154. doi: 10.1016/j.tig.2004.01.008

Li, X. J., Li, S. H., Sharp, A. H., Nucifora, F. C. Jr., Schilling, G., et al. (1995). A huntingtin-associated protein enriched in brain with implications for pathology. Nature 378, 398-402. doi: 10.1038/378398a0

Liou, J., Kim, M. L., Heo, W. D., Jones, J. T., Myers, J. W., Ferrell, J. E., et al. (2005). STIM is a Ca2+ sensor essential for Ca2+-store-depletiontriggered Ca2+ influx. Curr. Biol. 15, 1235-1241. doi: 10.1016/j.cub.2005. 05.055

Luo, W. J., Wang, H., Li, H., Kim, B. S., Shah, S., Lee, H. J., et al. (2003). PEN-2 and APH-1 coordinately regulate proteolytic processing of presenilin 1. J. Biol. Chem. 278, 7850-7854. doi: 10.1074/jbc.C200648200

Luthi-Carter, R., Hanson, S. A., Strand, A. D., Bergstrom, D. A., Chun, W., Peters, N. L., et al. (2002a). Dysregulation of gene expression in the R6/2 model of polyglutamine disease: parallel changes in muscle and brain. Hum. Mol. Genet. 11, 1911-1926. doi: 10.1093/hmg/11.17.1911

Luthi-Carter, R., Strand, A. D., Hanson, S. A., Kooperberg, C., Schilling, G., La Spada, A. R., et al. (2002b). Polyglutamine and transcription: gene expression changes shared by DRPLA and Huntington's disease mouse models reveal context-independent effects. Hum. Mol. Genet. 11, 1927-1937. doi: 10.1093/hmg/11.17.1927

Luthi-Carter, R., Strand, A., Peters, N. L., Solano, S. M., Hollingsworth, Z. R., Menon, A. S., et al. (2000). Decreased expression of striatal signaling genes in a mouse model of Huntington's disease. Hum. Mol. Genet. 9, 1259-1271. doi: 10.1093/hmg/9.9.1259

Mangiarini, L., Sathasivam, K., Seller, M., Cozens, B., Harper, A., Hetherington, C., et al. (1996). Exon 1 of the HD gene with an expanded CAG repeat is sufficient to cause a progressive neurological phenotype in transgenic mice. Cell 87, 493-506. doi: 10.1016/S0092-8674(00)81369-0

Massouh, M., Wallman, M. J., Pourcher, E., and Parent, A. (2008). The fate of the large striatal interneurons expressing calretinin in Huntington's disease. Neurosci. Res. 62, 216-224. doi: 10.1016/j.neures.2008.08.007

Matsuzawa, S. I., and Reed, J. C. (2001). Siah-1, SIP, and Ebi collaborate in a novel pathway for beta-catenin degradation linked to p53 responses. Mol. Cell 7, 915-926. doi: 10.1016/S1097-2765(01)00242-8

Michalak, M., Groenendyk, J., Szabo, E., Gold, L. I., and Opas, M. (2009). Calreticulin, a multi-process calcium-buffering chaperone of the endoplasmic reticulum. Biochem. J. 417, 651-666. doi: 10.1042/BJ20081847

Moores, J. N., Roy, S., Nicholson, D. W., and Staveley, B. E. (2008). Huntingtin interacting protein 1 can regulate neurogenesis in Drosophila. Eur. J. Neurosci. 28, 599-609. doi: 10.1111/j.1460-9568.2008.06359.x

Ng, A. N., Krogh, M., and Toresson, H. (2011). Dendritic EGFP-STIM1 activation after type I metabotropic glutamate and muscarinic acetylcholine receptor stimulation in hippocampal neuron. J. Neurosci. Res. 89, 1235-1244. doi: $10.1002 /$ jnr.22648

Nikoletopoulou, V., and Tavernarakis, N. (2012). Calcium homeostasis in aging neurons. Front. Genet. 3:200. doi: 10.3389/fgene.2012.00200

Noble, W. S. (2009). How does multiple testing correction work? Nat. Biotechnol. 27, 1135-1137. doi: 10.1038/nbt1209-1135

Park, C. Y., Shcheglovitov, A., and Dolmetsch, R. (2010). The CRAC channel activator STIM1 binds and inhibits L-type voltage-gated calcium channels. Science 330, 101-105. doi: 10.1126/science.1191027

Paxinos, G., and Franklin, K. B. J. (2004). The Mouse Brain in Stereotaxic Coordinates. San Diego, CA: Academic Press.

Puzianowska-Kuznicka, M., and Kuznicki, J. (2009). The ER and ageing II: calcium homeostasis. Ageing Res. Rev. 8, 160-172. doi: 10.1016/j.arr.2009.05.002

Ribeiro, F. M., Paquet, M., Ferreira, L. T., Cregan, T., Swan, P., Cregan, S. P., et al. (2010). Metabotropic glutamate receptor-mediated cell signaling pathways are altered in a mouse model of Huntington's disease. J. Neurosci. 30, 316-324. doi: 10.1523/JNEUROSCI.4974-09.2010

Rockabrand, E., Slepko, N., Pantalone, A., Nukala, V. N., Kazantsev, A., Marsh, J. L., et al. (2007). The first 17 amino acids of Huntingtin modulate its sub-cellular localization, aggregation and effects on calcium homeostasis. Hum. Mol. Genet. 16, 61-77. doi: 10.1093/hmg/ dd1 440

Roos, J., DiGregorio, P. J., Yeromin, A. V., Ohlsen, K., Lioudyno, M., Zhang, S., et al. (2005). STIM1, an essential and conserved component of store-operated Ca2+ channel function. J. Cell Biol. 169, 435-445. doi: 10.1083/jcb.2005 02019

Ryazantseva, M., Skobeleva, K., and Kaznacheyeva, E. (2013). Familial Alzheimer's disease-linked presenilin-1 mutation M146V affects store-operated calcium entry: does gain look like loss? Biochimie 95, 1506-1509. doi: 10.1016/j.biochi.2013.04.009

Seto-Ohshima, A., Emson, P. C., Lawson, E., Mountjoy, C. Q., and Carrasco, L. H. (1988). Loss of matrix calcium-binding protein-containing neurons in Huntington's disease. Lancet 1, 1252-1255. doi: 10.1016/S0140-6736(88) 92073-9

Sharma, S., Quintana, A., Findlay, G. M., Mettlen, M., Baust, B., Jain, M., et al. (2013). An siRNA screen for NFAT activation identifies septins as coordinators of store-operated Ca2+ entry. Nature 499, 238-242. doi: 10.1038/nature12229

Skibinska-Kijek, A., Wisniewska, M. B., Gruszczynska-Biegala, J., Methner, A., and Kuznicki, J. (2009). Immunolocalization of STIM1 in the mouse brain. Acta Neurobiol. Exp. (Wars) 69, 413-428.

Slow, E. J., van Raamsdonk, J., Rogers, D., Coleman, S. H., Graham, R. K., Deng, Y., et al. (2003). Selective striatal neuronal loss in a YAC128 mouse model of Huntington disease. Hum. Mol. Genet. 12, 1555-1567. doi: $10.1093 / \mathrm{hmg} / \mathrm{ddg} 169$

Steinbeck, J. A., Henke, N., Opatz, J., Gruszczynska-Biegala, J., Schneider, L., Theiss, S., et al. (2011). Store-operated calcium entry modulates neuronal network activity in a model of chronic epilepsy. Exp. Neurol. 232, 185-194. doi: 10.1016/j.expneurol.2011.08.022

Sugars, K. L., and Rubinsztein, D. C. (2003). Transcriptional abnormalities in Huntington disease. Trends Genet. 19, 233-238. doi: 10.1016/S01689525(03)00074-X

Supnet, C., and Bezprozvanny, I. (2011). Presenilins function in ER calcium leak and Alzheimer's disease pathogenesis. Cell Calcium 50, 303-309. doi: 10.1016/j.ceca.2011.05.013

Tang, T. S., Slow, E., Lupu, V., Stavrovskaya, I. G., Sugimori, M., Llinas, R., et al. (2005). Disturbed Ca2+ signaling and apoptosis of medium spiny neurons in Huntington's disease. Proc. Natl. Acad. Sci. U.S.A. 102, 2602-2607. doi: 10.1073/pnas.0409402102

Tang, T. S., Tu, H., Chan, E. Y., Maximov, A., Wang, Z., Wellington, C. L., et al. (2003). Huntingtin and huntingtin-associated protein 1 influence neuronal calcium signaling mediated by inositol- $(1,4,5)$ triphosphate receptor type 1 . Neuron 39, 227-239. doi: 10.1016/S0896-6273(03)00366-0

Tang, T. S., Tu, H., Orban, P. C., Chan, E. Y., Hayden, M. R., and Bezprozvanny, I. (2004). HAP1 facilitates effects of mutant huntingtin on inositol 1,4,5trisphosphate-induced $\mathrm{Ca}$ release in primary culture of striatal medium spiny neurons. Eur. J. Neurosci. 20, 1779-1787. doi: 10.1111/j.1460-9568.2004. 03633.x

Tarlac, V., and Storey, E. (2003). Role of proteolysis in polyglutamine disorders. J. Neurosci. Res. 74, 406-416. doi: 10.1002/jnr.10746

Toescu, E. C., and Verkhratsky, A. (2007). The importance of being subtle: small changes in calcium homeostasis control cognitive decline in normal aging. Aging Cell 6, 267-273. doi: 10.1111/j.1474-9726.2007.00296.x

Van Raamsdonk, J. M., Warby, S. C., and Hayden, M. R. (2007). Selective degeneration in YAC mouse models of Huntington disease. Brain Res. Bull. 72, 124-131. doi: 10.1016/j.brainresbull.2006.10.018

van Roon-Mom, W. M., Pepers, B. A., t Hoen, P. A., Verwijmeren, C. A., den Dunnen, J. T., Dorsman, J. C., et al. (2008). Mutant huntingtin activates Nrf2-responsive genes and impairs dopamine synthesis in a PC12 model of Huntington's disease. BMC Mol. Biol. 9:84. doi: 10.1186/1471-2199-9-84

Vig, M., Peinelt, C., Beck, A., Koomoa, D. L., Rabah, D., Koblan-Huberson, M., et al. (2006). CRACM1 is a plasma membrane protein essential for store-operated Ca2+ entry. Science 312, 1220-1223. doi: 10.1126/science.1127883

Vonsattel, J. P., Myers, R. H., Stevens, T. J., Ferrante, R. J., Bird, E. D., and Richardson, E. P. Jr. (1985). Neuropathological classification of Huntington's disease. J. Neuropathol. Exp. Neurol. 44, 559-577. doi: 10.1097/00005072198511000-00003

Winsky, L., and Kuznicki, J. (1996). Antibody recognition of calcium-binding proteins depends on their calcium-binding status. J. Neurochem. 66, 764-771. doi: 10.1046/j.1471-4159.1996.66020764.x 
Wojda, U., Salinska, E., and Kuznicki, J. (2008). Calcium ions in neuronal degeneration. IUBMB Life 60, 575-590. doi: 10.1002/iub.91

Wu, J., Shih, H. P., Vigont, V., Hrdlicka, L., Diggins, L., Singh, C., et al. (2011). Neuronal store-operated calcium entry pathway as a novel therapeutic target for Huntington's disease treatment. Chem. Biol. 18, 777-793. doi: 10.1016/j.chembiol.2011.04.012

Wu, L. L., and Zhou, X. F. (2009). Huntingtin associated protein 1 and its functions. Cell Adh. Migr. 3, 71-76. doi: 10.4161/cam.3.1.7511

Zeron, M. M., Hansson, O., Chen, N., Wellington, C. L., Leavitt, B. R., Brundin, P., et al. (2002). Increased sensitivity to N-methyl-D-aspartate receptor-mediated excitotoxicity in a mouse model of Huntington's disease. Neuron 33, 849-860. doi: 10.1016/S0896-6273(02)00615-3

Zucker, B., Luthi-Carter, R., Kama, J. A., Dunah, A. W., Stern, E. A., Fox, J. H., et al. (2005). Transcriptional dysregulation in striatal projection- and interneurons in a mouse model of Huntington's disease: neuronal selectivity and potential neuroprotective role of HAP1. Hum. Mol. Genet. 14, 179-189. doi: $10.1093 / \mathrm{hmg} / \mathrm{ddi} 014$
Conflict of Interest Statement: The authors declare that the research was conducted in the absence of any commercial or financial relationships that could be construed as a potential conflict of interest.

Received: 18 July 2013; accepted: 05 November 2013; published online: 25 November 2013.

Citation: Czeredys M, Gruszczynska-Biegala J, Schacht T, Methner A and Kuznicki J (2013) Expression of genes encoding the calcium signalosome in cellular and transgenic models of Huntington's disease. Front. Mol. Neurosci. 6:42. doi: 10.3389/fnmol. 2013.00042

This article was submitted to the journal Frontiers in Molecular Neuroscience.

Copyright $\odot 2013$ Czeredys, Gruszczynska-Biegala, Schacht, Methner and Kuznicki.

This is an open-access article distributed under the terms of the Creative Commons Attribution License (CC BY). The use, distribution or reproduction in other forums is permitted, provided the original author(s) or licensor are credited and that the original publication in this journal is cited, in accordance with accepted academic practice. No use, distribution or reproduction is permitted which does not comply with these terms. 